\title{
THE MOMENTS OF THE DISCOUNTED LOSS AND THE DISCOUNTED DIVIDENDS FOR A SPECTRALLY NEGATIVE LÉVY RISK PROCESS
}

\author{
ESTHER FROSTIG, ${ }^{*}$ University of Haifa
}

\begin{abstract}
Consider a spectrally negative risk process where, on ruin, the deficit is immediately paid, and the process restarts from 0 . When the process reaches a threshold $b$, all the surplus above $b$ is paid as dividend. Applying the theory of exit times for a spectrally negative Lévy process and its reflection at the maximum and at the minimum, we obtain recursive formulae for the following moments. (i) The moments of the discounted loss until the process reaches $b$. This is equivalent to the moments of the discounted dividends in the dual model under the barrier strategy. (ii) The moments of the discounted loss for models with and without a dividend barrier for the infinite horizon. (iii) The moments of the discounted dividends for the infinite horizon.
\end{abstract}

Keywords: Dividends; barrier strategy; capital injection; exit times; reflected process; scale function; dual model

2010 Mathematics Subject Classification: Primary 60G51

Secondary 91B30

\section{Introduction}

The expected discounted dividends paid to shareholders is one of the performance measures in risk theory. The most studied strategy is the barrier strategy with parameter $b$. Under this strategy, no dividends are paid while the surplus is below $b$, and all the overflow above $b$ is paid as dividend. Dividends are paid until ruin occurs, i.e. until the first time that the surplus is negative. De Finetti (1957) introduced the dividend model. When the risk process evolves as a random walk in discrete time with steps \pm 1 , he proved that the barrier strategy is optimal. The optimality of this strategy was also proved when the risk process evolves as a Brownian motion with drift, see Asmussen and Taksar (1997) and Asmussen et al. (2000), and for the Cramer-Lundberg risk process with exponentially distributed claims, see Gerber (1969) and also Schmidli (2008, Chapter 2).

In the recent years researchers have modelled the surplus of an insurance company as a spectrally negative Lévy process, i.e. a process with stationary independent increments and without positive jumps. In the sequel, we will call this model the primal model. For this model, Avram et al. (2007) proved the optimality of the barrier strategy under some conditions on the generator of the Lévy process. For more explicit conditions on the Lévy measure, see Loeffen (2008).

Another model that has been studied lately is the dual risk model. In this model the surplus of a company is described as a Lévy process without negative jumps. This model is appropriate to describe the surplus of companies that specialize in inventions and discoveries. The barrier

Received 24 January 2014; revision received 3 September 2014.

* Postal address: Department of Statistics, University of Haifa, Haifa, 31905, Israel.

Email address: frostig@stat.haifa.ac.il 
strategy for dividends in the dual model was considered by Avanzi et al. (2007) for the compound Poisson dual model, and by Avanzi and Gerber (2008) for the compound Poisson dual model perturbed by diffusion.

Renaud and Zhou (2007) and Kyprianou and Palmowski (2007) obtained the moments of the discounted dividends until ruin, when the dividends are paid according to the barrier strategy. Renaud and Zhou's proof is based on some basic ideas from fluctuation theory, while Kyprianou and Palmowski's proof is based on excursion theory. They derived an elegant recursive formula for the moments using recursion.

Lately, Cheung and Drekic (2008) considered the dual model, where gains arrive according to a Poisson process. They obtained differential equations for the $n$th moment as a function of lower-order moments and obtained an explicit solution when the gains have mixed exponential distribution.

Since under the barrier strategy ruin occurs with probability 1, Dickson and Waters (2004) considered models where the shareholders receive dividends according to the barrier strategy but also pay the deficit whenever ruin occurs. Dickson and Waters obtained the expected discounted dividends and the expected discounted deficit paid for the compound Poisson risk model. Avram et al. (2007) considered such a model but for spectrally negative Lévy risk processes. Kulenko and Schmidli (2008) and Avram et al. (2007) studied the optimal dividend policy when the deficit is recovered whenever ruin occurs. Kulenko and Schmidli (2008) considered the compound Poisson risk model and proved the optimality of the barrier strategy under some conditions. Avram et al. (2007) proved under some conditions the optimality of the barrier strategy for general spectrally negative Lévy processes; see also Loeffen (2008).

Avanzi et al. (2011) considered the dual model with capital injection, and proved the optimality of the barrier strategy in the dual model in the case of a compound Poisson risk process with exponentially distributed jumps. Bayrakatar et al. (2013) proved the optimality of the barrier strategy for a generally Lévy process without negative jumps.

Consider the spectrally negative Lévy process $X$ with dividend barrier $b$ and recoveries. Assume that at time 0 the surplus is $u$. Consider the discount dividend payments and the discounted deficit payments. Consider the dual model $b-X$. Clearly, the dividends in the primal model with initial reserve $u$ are the same as the deficit payments in the dual model with initial reserve $b-u$, and the deficit payments in the primal model are the same as the dividend payments in the dual model.

This paper is motivated by Renaud and Zhou (2007) and Kyprianou and Palmowski (2007). Our objective is to obtain recursive formulae for the moments of the discounted deficit payment and the discounted dividend payments for the spectrally negative Lévy risk model with dividend payments and recoveries.

The outline of this paper is as follows. In the next section we present some results on the exit times for a spectrally negative Lévy process and its reflection at the infimum and at the supremum. In Section 3 we obtain a recursive formula for the expected discounted loss until the process reaches level $b$. In Section 4 we consider the expected discounted loss for the infinite horizon for a risk process where dividends are paid according to the barrier strategy and the deficit is always recovered. In Section 5 we obtain a recursive formula for the moments of the discounted dividends for the model where the dividends are paid according to the barrier strategy and the deficit is recovered. 


\section{Preliminaries}

\subsection{Spectrally negative Lévy risk processes}

Let $\left(X_{t}, t \geq 0\right)$ be a spectrally negative Lévy risk process. Throughout this paper, we denote by $\mathbb{P}_{x}, \mathbb{E}_{x}$ the probability or expectation given that $X_{0}=x$. When $X_{0}=0$, we simply write $\mathbb{P}, \mathbb{E}$. The Laplace transform of $X_{t}$ exists for $\theta \geq 0$ and is given by

$$
\mathbb{E}\left[\mathrm{e}^{\theta X_{t}}\right]=\mathrm{e}^{t \psi(\theta)},
$$

where

$$
\psi(\theta)=\mathrm{d} \theta+\frac{1}{2} \sigma^{2} \theta^{2}+\int_{-\infty}^{0}\left(\mathrm{e}^{\theta z}-1-\theta z \mathbf{1}_{(-1,0)}(z)\right) \Pi(\mathrm{d} z),
$$

and $\mathbf{1}_{A}(z)$ is the indicator function of a set $A$, and it is 1 if $z \in A$ and 0 otherwise. We have $d \in \mathbb{R}, \sigma \geq 0$, and $\Pi$ is a $\sigma$-finite measure on $(-\infty, 0)$, where

$$
\int_{-\infty}^{0}\left(1 \wedge z^{2}\right) \Pi(\mathrm{d} z)<\infty .
$$

We exclude processes with monotone paths. When the process has a bounded variation its Laplace exponent is

$$
\psi(\theta)=c \theta+\int_{-\infty}^{0}\left(\mathrm{e}^{\theta z}-1\right) \Pi(\mathrm{d} z)
$$

where $c=d-\int_{-1}^{0} x \Pi(\mathrm{d} x), \psi(\theta)$ is strictly convex, and $\lim _{\theta \rightarrow \infty} \psi(\theta)=\infty$. Thus, there exists a function $\Phi(\theta)=\sup \{x \geq 0: \psi(x)=\theta\}$, and $\Phi(\psi(\theta))=\theta, \theta \geq 0$.

\subsection{Exit times}

Let

$$
T_{b}^{+}=\inf \left\{t \geq 0: X_{t} \geq b\right\}, \quad T_{a}^{-}=\inf \left\{t \geq 0: X_{t} \leq a\right\},
$$

where the convention is inf $\varnothing=\infty$. For $a<b$, let

$$
T_{(a, b)}=\min \left(T_{b}^{+}, T_{a}^{-}\right)=\inf \left\{t: X_{t} \notin(a, b)\right\} .
$$

For $q \geq 0$, there exists a unique continuous function $W^{(q)}$ called a $q$-scale function defined by its Laplace transform as

$$
\int_{0}^{\infty} \mathrm{e}^{-\theta x} W^{(q)}(x) \mathrm{d} x=\frac{1}{\psi(\theta)-q}, \quad \theta>\Phi(q) .
$$

For $q=0$, we write $W^{(0)}=W$, where $W$ is called the scale function. It holds that $W^{(q)}(x)=0$ for $x<0$.

Another function related to the scale function is $Z^{(q)}$ given by

$$
Z^{(q)}(x)=1+q \int_{0}^{x} W^{(q)}(y) \mathrm{d} y .
$$

The following properties of $W^{(q)}(x)$ will be useful.

(P1) $W^{(q)}(0)=0$ if and only if $X$ has unbounded variation. Otherwise, it is equal to $1 / c$, where $c>0$ is the drift; see Kyprianou (2006, Lemma 8.6). 
(P2) The left and right derivatives of $W^{(q)}(x)$ exist for $x \in(0, \infty)$ and are denoted by $W_{-}^{(q)^{\prime}}$ and $W_{+}^{(q)^{\prime}}$, respectively; see Kyprianou (2006, Lemma 8.2) and Kuznetsov et al. $(2012$, Lemma 2.3). If the paths of $X$ are of unbounded variation, or when $\Pi(-\infty,-x)$ is continuous, then the derivative $W^{(q)^{\prime}}(x)$ exists and it is continuous for all $x>0$; see Kuznetsov et al. (2012, Lemma 2.3).

(P3) It holds that

$$
W_{+}^{(q)^{\prime}}(0)=\lim _{\varepsilon \rightarrow 0+} \frac{W^{(q)}(\varepsilon)-W^{(q)}(0)}{\varepsilon} .
$$

When $\sigma>0, W_{+}^{(q)^{\prime}}(0)=2 / \sigma^{2}$; see Pistorius (2004, Lemma 4) and Kuznetsov et al. (2012, Lemma 3.2).

(P4) When $\sigma>0$,

$$
\lim _{\varepsilon \rightarrow 0} W_{+}^{(q)^{\prime}}(\varepsilon)=W_{+}^{(q)^{\prime}}(0)=\frac{2}{\sigma^{2}} .
$$

This result can be proved directly by applying (P3), or by taking the limit as $x \rightarrow 0$ of Kusnetsov et al. (2012, Equation (65)).

The solution of the one-sided and two-sided exit problems for a spectrally negative Lévy process is given in terms of the scale function.

Theorem 2.1. (Kyprianou (2006, Theorem 8.1.)) (i) For $0<x \leq b$ and $q \geq 0$,

$$
\mathbb{E}_{x}\left[\mathrm{e}^{-q T_{b}^{+}} \mathbf{1}_{\left\{T_{b}^{+}<T_{0}^{-}\right\}}\right]=\frac{W^{(q)}(x)}{W^{(q)}(b)} .
$$

(ii) For $0<x \leq b$ and $q \geq 0$,

$$
\mathbb{E}_{x}\left[\mathrm{e}^{-q T_{0}^{-}} \mathbf{1}_{\left\{T_{0}^{-}<T_{b}^{+}\right\}}\right]=Z^{(q)}(x)-Z^{(q)}(b) \frac{W^{(q)}(x)}{W^{(q)}(b)} .
$$

Scale functions are used to describe the so-called potential measure associated with the one-sided or two-sided exit problems. For $x, y \in[0, b]$, we are interested in

$$
U^{(q)}(x, \mathrm{~d} y)=\int_{0}^{\infty} \mathrm{e}^{-q t} \mathbb{P}_{x}\left(X_{t} \in \mathrm{d} y, T_{(0, b)}>t\right) \mathrm{d} t .
$$

Here, $U^{(q)}(x, \mathrm{~d} y)$ (with $U^{(0)}=U$ ) is the $q$-potential measure of a spectrally negative Lévy process $X_{t}$ killed on exiting $[0, b]$, where $x, y \in[0, b]$.

Theorem 2.2. (Suprun (1976) and Bertoin (1997)) It holds that $U^{(q)}(x, \mathrm{~d} y)$ has a density $u^{(q)}(x, y)$ given by

$$
u^{(q)}(x, y)=\frac{W^{(q)}(x) W^{(q)}(b-y)}{W^{(q)}(b)}-W^{(q)}(x-y) .
$$

For $u<x<b$ and $\sigma>0$, the following equality holds (see, e.g. Mijatović and Pistorius (2012, Equation (2.6))):

$$
\mathbb{E}_{x}\left[\mathrm{e}^{-q T_{(u, b)}} \mathbf{1}_{\left\{X_{T_{(u, b)}}=u\right\}}\right]=\frac{\sigma^{2}}{2}\left(W_{+}^{(q)^{\prime}}(x-u)-\frac{W_{+}^{(q)^{\prime}}(b-u)}{W^{(q)}(b-u)} W^{(q)}(x-u)\right) .
$$


In the sequel, we apply also the results related to the reflected Lévy processes at the infimum and at the supremum. Let $S_{t}=\sup _{0 \leq s \leq t}\left(X_{s} \vee 0\right)$ and let $I_{t}=\inf _{0 \leq s \leq t}\left(X_{t} \wedge 0\right)$, where $c \vee d=\max (c, d)$ and $c \wedge d=\min (c, d)$. Let $Y=X-I$ and $\hat{Y}=S-X$ denote, respectively, the Lévy process reflected at its past infimum $I$, and at its past supremum $S$. Let $\tau_{b}$ be the first entrance time of $Y$ to $(b, \infty)$, and $\hat{\tau}_{b}$ the first entrance time of $\hat{Y}$ to $(b, \infty), b>0$. The Laplace transform of $\tau_{b}$ and $\hat{\tau}_{b}$ can be expressed in terms of $W^{(q)}$ and $Z^{(q)}$ as

$$
\begin{aligned}
\mathbb{E}_{x}\left[\mathrm{e}^{-q \tau_{b}}\right] & =\frac{Z^{(q)}(x)}{Z^{(q)}(b)}, \\
\mathbb{E}_{-x}\left[\mathrm{e}^{-q \hat{\tau}_{b}}\right] & =Z^{(q)}(b-x)-q W^{(q)}(b-x) \frac{W^{(q)}(b)}{W_{+}^{(q)^{\prime}}(b)} \quad \text { for } x \in[0, b]
\end{aligned}
$$

see Pistorius (2004, Proposition 2). Note that when $\hat{Y}_{0}=x>0, X_{0}=-x$. Let $A \subseteq[0, b]$. Similar to (2.6), we define

$$
\begin{aligned}
& R^{q}(x, A)=\int_{0}^{\infty} \mathbb{P}\left(Y_{t} \in A, t<\tau_{b}\right) \mathrm{e}^{-q t} \mathrm{~d} t, \\
& \hat{R}^{q}(x, A)=\int_{0}^{\infty} \mathbb{P}\left(\hat{Y}_{t} \in A, t<\hat{\tau}_{b}\right) \mathrm{e}^{-q t} \mathrm{~d} t .
\end{aligned}
$$

Pistorius (2004, Theorem 1) proved the following theorem.

Theorem 2.3. (i) The measure $R^{q}(x, \cdot)$ is absolutely continuous with respect to the Lebesgue measure and $a$ version of its density is given by

$$
r^{q}(x, y)=\frac{Z^{(q)}(x)}{Z^{(q)}(b)} W^{(q)}(b-y)-W^{(q)}(x-y), \quad x, y \in[0, b) .
$$

(ii) Let $\hat{r}(x, 0)=W^{(q)}(b-x) W^{(q)}(0) / W_{+}^{(q)^{\prime}}(b)$ for $x \geq 0$ and set

$$
\hat{r}^{q}(x, y)=W^{(q)}(b-x) \frac{W_{+}^{(q)^{\prime}}(y)}{W_{+}^{(q)^{\prime}}(b)}-W^{(q)}(y-x), \quad x, y \in[0, b), y \neq 0 .
$$

Then, $\hat{r}^{q}(x, 0) \delta_{0}(\mathrm{~d} y)+\hat{r}^{q}(x, y) \mathrm{d} y$ is a version of the measure $\hat{R}^{(q)}(x, \mathrm{~d} y)$.

\section{Moments of the discounted loss until reaching $b$, or moments of the discounted dividends in the dual model}

Consider a spectrally negative Lévy risk process as described by (2.1) and (2.2). Assume that whenever ruin occurs, i.e. the process down-crosses 0 , the deficit is paid by the shareholders. We are interested in the moments of the discounted loss (deficit) until the process reaches the level $b>0$.

Given that $X_{0}=u$, the discounted loss until the process reaches $b$ is the same as the discounted dividends, according to the barrier strategy in the dual model starting at $b-u$.

Throughout, we denote by $q$ the discount factor. Let $L_{t}$ be the loss until time $t$. Note that

$$
L_{t}=-I_{t} .
$$

The modified risk process with recoveries behaves as the reflected process at the infimum $Y=X-I$. Note that when $X_{0}=u>0, I_{0}=0$, the process $Y$ behaves as $X$ until time $T_{0}^{-}$, 
the first entrance time of $X_{t}$ to $(-\infty, 0)$. Let $L^{b}(u)=L_{\tau_{b}}$ be the discounted loss until the modified process $Y$ reaches $b$ when $X_{0}=Y_{0}=u$. For $0 \leq u \leq b$, let

$$
\ell_{n}(u, b)=\mathbb{E}_{u}\left[\left(\int_{0}^{\tau_{b}} \mathrm{e}^{-q t} \mathrm{~d} L_{t}\right)^{n}\right]
$$

In Sections 3 and 4, we assume that the following assumption holds.

Assumption 3.1. Assume that

$$
\int_{z=0}^{\infty} z^{i} \Pi(-\mathrm{d} z)<\infty
$$

whenever such an integral appears.

Remark 3.1. Assumption 3.1 implies that the risk process is the sum of an independent Brownian motion with variance $\sigma^{2} \geq 0$, and a bounded variation Lévy process with negative jumps. Thus, the only source of unbounded variation is due to the Brownian motion component.

\subsection{Bounded variation case, $u=0$}

In this section we obtain $\ell_{n}(0, b)$ for the bounded variation case, i.e. $\sigma=0$. Define $\ell_{0}(0, b)=1$.

Proposition 3.1. It holds that

$$
\ell_{n}(0, b)=\frac{1}{Z^{(n q)}(b)} \sum_{i=1}^{n}\left(\begin{array}{l}
n \\
i
\end{array}\right) \int_{z=0}^{\infty} z^{i} \int_{y=0}^{b} W^{(n q)}(b-y) \Pi(-y-\mathrm{d} z) \mathrm{d} y \ell_{n-i}(0, b) .
$$

Proof. Since Assumption 3.1 holds the right-hand side of (3.1) is finite, as well as similar expressions in the rest of this section. Denote by $L^{b}(0)$ the discounted loss until the process hits $b$ given that $X_{0}=0$. We have

$$
L^{b}(0)=\mathbf{1}_{\left\{T_{0}^{-}<T_{b}^{+}\right\}} \mathrm{e}^{-q T_{0}^{-}}\left(\left|X_{T_{0}^{-}}\right|+\tilde{L}^{b}(0)\right),
$$

where $\tilde{L}^{b}(0)$ is the discounted loss until reaching $b$ from the moment of the first recovery. From the strong Markov property, $\tilde{L}^{b}(0)$ is independent of $T_{0}^{-}$and of $X_{T_{0}^{-}}$and has the same distribution as $L^{b}(0)$. Taking the $n$th power of (3.2) yields

$$
\left(L^{b}(0)\right)^{n}=\mathbf{1}_{\left\{T_{0}^{-}<T_{b}^{+}\right\}} \mathrm{e}^{-n q T_{0}^{-}} \sum_{i=0}^{n}\left(\begin{array}{l}
n \\
i
\end{array}\right)\left(\left|X_{T_{0}^{-}}\right|\right)^{i}\left(\tilde{L}^{b}(0)\right)^{n-i} .
$$

Taking the expectation of both sides of (3.3) and applying (2.6) and (2.7), we obtain

$$
\begin{aligned}
\ell_{n}(0, b) & =\sum_{i=0}^{n}\left(\begin{array}{l}
n \\
i
\end{array}\right) \int_{z=0}^{\infty} z^{i} \int_{y=0}^{b} \mathrm{e}^{-n q t} \mathbb{P}\left(T_{(0, b)}>t, X_{t} \in \mathrm{d} y\right) \Pi(-y-\mathrm{d} z) \mathrm{d} y \ell_{n-i}(0, b) \\
& =\sum_{i=0}^{n}\left(\begin{array}{l}
n \\
i
\end{array}\right) \int_{z=0}^{\infty} z^{i} \int_{y=0}^{b} u^{(n q)}(0, y) \Pi(-y-\mathrm{d} z) \mathrm{d} y \ell_{n-i}(0, b) .
\end{aligned}
$$


Note that, by (2.5),

$$
\begin{aligned}
\int_{z=0}^{\infty} \int_{y=0}^{b} u^{(n q)}(0, y) \Pi(-y-\mathrm{d} z) \mathrm{d} y & =\mathbb{E}_{0}\left[\mathrm{e}^{-n q T_{0}^{-}} \mathbf{1}_{\left\{T_{0}^{-}<T_{b}\right\}}\right] \\
& =Z^{(n q)}(0)-\frac{Z^{(n q)}(b) W^{(n q)}(0)}{W^{(n q)}(b)} .
\end{aligned}
$$

Substituting (2.7) and (3.5) into (3.4), and since $W^{(n q)}(y)=0$ for $y<0$, and $Z^{(n q)}(0)=1$, we obtain

$$
\begin{aligned}
\ell_{n}(0, b) & \frac{Z^{(n q)}(b) W^{(n q)}(0)}{W^{(n q)}(b)} \\
= & \sum_{i=1}^{n}\left(\begin{array}{l}
n \\
i
\end{array}\right) \int_{z=0}^{\infty} z^{i} \int_{y=0}^{b} \frac{W^{(n q)}(0) W^{(n q)}(b-y)}{W^{(n q)}(b)} \Pi(-y-\mathrm{d} z) \mathrm{d} y \ell_{n-i}(0, b) .
\end{aligned}
$$

In the bounded variation case $W^{(n q)}(0)=1 / c>0$. Dividing both sides of the last equation by $Z^{(n q)}(b) W^{(n q)}(0) / W^{(n q)}(b)$ yields (3.1).

\subsection{Unbounded variation case, $u=0$}

Due to Assumption 3.1 and Remark 3.1, the only source of unbounded variation is due to the Brownian motion, thus in this section, we assume that $\sigma>0$.

Proposition 3.2. Assume that $\sigma>0$. Then, for $n \geq 1$,

$$
\begin{aligned}
\ell_{n}(0, b)=\frac{1}{Z^{(n q)}(b)} \sum_{i=1}^{n}\left(\begin{array}{l}
n \\
i
\end{array}\right) \int_{z=0}^{\infty} z^{i} \int_{y=0}^{b} & W^{(n q)}(b-y) \Pi(-y-\mathrm{d} z) \mathrm{d} y \ell_{n-i}(0, b) \\
& +n \frac{\sigma^{2}}{2} \frac{W^{(n q)}(b)}{Z^{(n q)}(b)} \ell_{n-1}(0, b) .
\end{aligned}
$$

Proof. We obtain lower and upper bounds for $\ell_{n}(0, b)$. Let $\varepsilon>0$. To obtain a lower bound, we consider the following strategy. The first deficit payment is done only when $X_{t}$ hits or down-crosses $-\varepsilon$ at time $T_{-\varepsilon}^{-}$. From that point the deficit is paid whenever it occurs until the process reaches $b$. Let $\underline{L}^{b, \varepsilon}$ be the discounted losses according to this policy. Since the deficit is discounted only from the moment it exceeds $\varepsilon$, we have $L^{b}(0) \geq \underline{L}^{b, \varepsilon}$ for $\varepsilon>0$. Then

$$
\underline{L}^{b, \varepsilon}=L_{1}^{b, \varepsilon}+\underline{L}_{2}^{b, \varepsilon}
$$

where

$$
\begin{gathered}
L_{1}^{b, \varepsilon}=\mathrm{e}^{-q T_{-\varepsilon}^{-}} \mathbf{1}_{\left\{X_{\left.T_{(-\varepsilon, b)}<-\varepsilon\right\}}\left(\left|X_{T_{(-\varepsilon, b)}}\right|+L^{b}(0)\right),\right.}, \\
\underline{L}_{2}^{b, \varepsilon}=\mathrm{e}^{-q T_{-\varepsilon}^{-}} \mathbf{1}_{\left\{X_{T_{(-\varepsilon, b)}}=-\varepsilon\right\}}\left(\varepsilon+L^{b}(0)\right) .
\end{gathered}
$$

Since

$$
\mathbf{1}_{\left\{X_{T_{(-\varepsilon, b)}}<-\varepsilon\right\}} \mathbf{1}_{\left\{X_{T_{(-\varepsilon, b)}}=-\varepsilon\right\}}=0,
$$

we obtain

$$
\left(\underline{L}^{b, \varepsilon}\right)^{n}=\left(L_{1}^{b, \varepsilon}\right)^{n}+\left(\underline{L}_{2}^{b, \varepsilon}\right)^{n} .
$$


From (3.7) and (3.8), we have

$$
\begin{gathered}
\left(L_{1}^{b, \varepsilon}\right)^{n}=\mathrm{e}^{-n q T_{-\varepsilon}^{-}} \mathbf{1}_{\left\{X_{T_{(-\varepsilon, b)}}<-\varepsilon\right\}} \sum_{i=0}^{n}\left(\begin{array}{c}
n \\
i
\end{array}\right)\left(\mid X_{\left.T_{(-\varepsilon, b)} \mid\right)^{i}\left(L^{b}(0)\right)^{n-i},}\right. \\
\left(\underline{L}_{2}^{b, \varepsilon}\right)^{n}=\mathrm{e}^{-n q T_{-\varepsilon}^{-}} \mathbf{1}_{\left\{X_{T_{(-\varepsilon, b)}}=-\varepsilon\right\}} \sum_{i=0}^{n}\left(\begin{array}{c}
n \\
i
\end{array}\right) \varepsilon^{i}\left(L^{b}(0)\right)^{n-i} .
\end{gathered}
$$

From (2.6)-(2.8) and by similar arguments leading to (3.4), it follows that

$$
\begin{aligned}
& \mathbb{E}_{0}\left[\left(L_{1}^{b, \varepsilon}\right)^{n}\right]=\sum_{i=0}^{n}\left(\begin{array}{l}
n \\
i
\end{array}\right) \int_{0}^{\infty} z^{i} \int_{0}^{b+\varepsilon} u^{(n q)}(\varepsilon, y) \Pi(-y-\mathrm{d} z) \mathrm{d} y \ell_{n-i}(0, b), \\
& \mathbb{E}_{0}\left[\left(\underline{L}_{2}^{b, \varepsilon}\right)^{n}\right]=\sum_{i=0}^{n}\left(\begin{array}{l}
n \\
i
\end{array}\right) \varepsilon^{i}\left(\frac{\sigma^{2}}{2} W^{(n q)^{\prime}}(\varepsilon)-\frac{W^{(n q)^{\prime}}(b+\varepsilon)}{W^{(n q)}(b+\varepsilon)} W^{(n q)}(\varepsilon)\right) \ell_{n-i}(0, b) .
\end{aligned}
$$

Properties (P1) and (P4) imply that for $i \geq 2$,

$$
\begin{gathered}
\varepsilon^{i} \frac{\sigma^{2}}{2}\left(W^{(n q)^{\prime}}(\varepsilon)-\frac{W^{(n q)^{\prime}}(b+\varepsilon)}{W^{(n q)}(b+\varepsilon)} W^{(n q)}(\varepsilon)\right)=o(\varepsilon), \\
\varepsilon \frac{\sigma^{2}}{2}\left(W^{(n q)^{\prime}}(\varepsilon)-\frac{W^{(n q)^{\prime}}(b+\varepsilon)}{W^{(n q)}(b+\varepsilon)} W^{(n q)}(\varepsilon)\right)=\varepsilon+o(\varepsilon) .
\end{gathered}
$$

Similar to the derivation of (3.5), from (2.5) and (2.6)-(2.8), it follows that

$$
\begin{aligned}
\mathbb{E}_{0}\left[\mathrm{e}^{-n q T_{-\varepsilon}^{-}} \mathbf{1}_{\left\{T_{-\varepsilon}^{-}<T_{b}^{+}\right\}}\right]= & Z^{(n q)}(\varepsilon)-Z^{(n q)}(b+\varepsilon) \frac{W^{(n q)}(\varepsilon)}{W^{(n q)}(b+\varepsilon)} \\
= & \int_{0}^{\infty} \int_{0}^{b+\varepsilon} u^{(n q)}(\varepsilon, y) \Pi(-y-\mathrm{d} z) \mathrm{d} y \\
& +\frac{\sigma^{2}}{2}\left(W^{(n q)^{\prime}}(\varepsilon)-\frac{W^{(n q)^{\prime}}(b+\varepsilon)}{W^{(n q)}(b+\varepsilon)} W^{(n q)}(\varepsilon)\right) .
\end{aligned}
$$

The second line of (3.14) is related to the case that the deficit at ruin is negative and the third line is the probability of reaching $-\varepsilon$ due to diffusion. Equations (3.10)-(3.14) imply that

$$
\begin{aligned}
\mathbb{E}\left[\underline{L}^{\varepsilon, b}\right]= & \mathbb{E}_{0}\left[\left(L_{1}^{b, \varepsilon}\right)^{n}\right]+\mathbb{E}_{0}\left[\left(\underline{L}_{2}^{b, \varepsilon}\right)^{n}\right] \\
= & \sum_{i=1}^{n}\left(\begin{array}{c}
n \\
i
\end{array}\right) \int_{0}^{\infty} z^{i} \int_{0}^{b+\varepsilon} u^{(n q)}(\varepsilon, y) \Pi(-y-\mathrm{d} z) \mathrm{d} y \ell_{n-i}(0, b)+n \varepsilon \ell_{n-1}(0, b) \\
& \quad+\left(Z^{(n q)}(\varepsilon)-Z^{(n q)}(b+\varepsilon) \frac{W^{(n q)}(\varepsilon)}{W^{(n q)}(b+\varepsilon)}\right) \ell_{n}(0, b)+o(\varepsilon) .
\end{aligned}
$$

To obtain an upper bound, assume that an amount of $\varepsilon$ is injected at time 0 . The losses are not recovered until the process reaches $-\varepsilon$ before reaching $b$. When the process reaches $-\varepsilon$ by creeping before hitting $b$, it restarts from 0 without deficit payment. If the process down-crosses $-\varepsilon$ by a jump then the deficit is paid and the process restarts from 0 . From that point the deficits 
are recovered as they occur. Let $\bar{L}^{b, \varepsilon}$ be the discounted loss under this regime. Since an amount $\varepsilon$ is paid in advance,

$$
L^{b}(0) \leq \bar{L}^{b, \varepsilon}, \quad \bar{L}^{b, \varepsilon}=\varepsilon+L_{1}^{b, \varepsilon}+\bar{L}_{2}^{b, \varepsilon},
$$

where

$$
\bar{L}_{2}^{b, \varepsilon}=\mathrm{e}^{-q T_{(-\varepsilon, b)}} \mathbf{1}_{\left\{X_{T_{(-\varepsilon, b)}}=-\varepsilon\right\}} L^{b}(0)
$$

From (3.9), we have

$$
\left(\bar{L}^{b, \varepsilon}\right)^{n}=\sum_{i=0}^{n}\left(\begin{array}{l}
n \\
i
\end{array}\right) \varepsilon^{i}\left(L_{1}^{b, \varepsilon}+\bar{L}_{2}^{b, \varepsilon}\right)^{n-i}=\sum_{i=0}^{n}\left(\begin{array}{l}
n \\
i
\end{array}\right) \varepsilon^{i}\left(\left(L_{1}^{b, \varepsilon}\right)^{n-i}+\left(\bar{L}_{2}^{b, \varepsilon}\right)^{n-i}\right) .
$$

Similarly to (3.10), it follows that

$$
\mathbb{E}_{0}\left[\left(L_{1}^{b, \varepsilon}\right)^{n-i}\right]=\sum_{j=0}^{n-i}\left(\begin{array}{c}
n-i \\
j
\end{array}\right) \int_{0}^{\infty} z^{j} \int_{0}^{b+\varepsilon} u^{((n-i) q)}(\varepsilon, y) \Pi(-y-\mathrm{d} z) \mathrm{d} y \ell_{n-i-j}(0, b),
$$

where, by (2.7),

$$
u^{((n-i) q)}(\varepsilon, y)=\frac{W^{((n-i) q)}(\varepsilon) W^{((n-i) q)}(b+\varepsilon-y)}{W^{((n-i) q)}(b+\varepsilon)}-W^{((n-i) q)}(\varepsilon-y) .
$$

Properties (P3) and (P4) of the $q$-scale function from Section 2.2 imply that, for $i \geq 1$,

$$
\varepsilon^{i} u^{((n-i) q)}(\varepsilon, y)=o(\varepsilon),
$$

and, therefore, since Assumption 3.1 holds

$$
\varepsilon^{i} \mathbb{E}_{0}\left[\left(L_{1}^{b, \varepsilon}\right)^{n-i}\right]=o(\varepsilon), \quad i \geq 1 .
$$

From (2.8) and the right continuity of $W^{(n q)^{\prime}}$ at 0 , we have

$$
\sum_{i=2}^{n}\left(\begin{array}{l}
n \\
i
\end{array}\right) \varepsilon^{i} \mathbb{E}\left[\mathrm{e}^{-(n-i) q T_{(-\varepsilon, b)}} \mathbf{1}_{X_{(-\varepsilon, b)}}\right] \ell_{n-i}(0, b)=o(\varepsilon) .
$$

Similarly, by properties (P2)-(P4),

$$
\begin{aligned}
\varepsilon \mathbb{E}\left[\mathrm{e}^{-(n-1) q T_{(-\varepsilon, b)}} \mathbf{1}_{\left.X_{T_{(-\varepsilon, b)}}\right]}\right. & =\varepsilon \frac{\sigma^{2}}{2}\left(W^{((n-1) q)^{\prime}}(\varepsilon)-\frac{W^{((n-1) q)^{\prime}}(b)}{W^{((n-1) q)}(b)} W^{((n-1) q)}(\varepsilon)\right) \\
& =\varepsilon+o(\varepsilon) .
\end{aligned}
$$

From (2.8), (3.16)-(3.19), and (3.12)-(3.14), we obtain

$$
\begin{aligned}
\mathbb{E}_{0}\left[\left(\bar{L}^{b, \varepsilon}\right)^{n}\right]=\sum_{i=1}^{n} & \left(\begin{array}{c}
n \\
i
\end{array}\right) \int_{0}^{\infty} z^{i} \int_{0}^{b+\varepsilon} u^{(n q)}(\varepsilon, y) \Pi(-y-\mathrm{d} z) \mathrm{d} y \ell_{n-i}(0, b) \\
& +n \varepsilon \ell_{n-1}(0, b)+o(\varepsilon) \\
& +\left(Z^{(n q)}(\varepsilon)-Z^{(n q)}(b+\varepsilon) \frac{W^{(n q)}(\varepsilon)}{W^{(n q)}(b+\varepsilon)}\right) \ell_{n}(0, b) .
\end{aligned}
$$


Since $\mathbb{E}_{0}\left[\left(\underline{L}^{b, \varepsilon}\right)^{n}\right] \leq \ell_{n}(0, b) \leq \mathbb{E}_{0}\left[\left(\bar{L}^{b, \varepsilon}\right)^{n}\right],(3.20)$ and (3.15) imply that up to $o(\varepsilon)$,

$$
\ell_{n}(0, b)=\mathbb{E}_{0}\left[\left(\underline{L}^{b, \varepsilon}\right)^{n}\right]=\mathbb{E}_{0}\left[\left(\bar{L}^{b, \varepsilon}\right)^{n}\right] .
$$

Solving for $\ell_{n}(0, b)$ yields

$$
\begin{aligned}
\ell_{n}(0, b) & \left(-n q \int_{0}^{\varepsilon} W^{(n q)}(x) \mathrm{d} x+Z^{(n q)}(b+\varepsilon) \frac{W^{(n q)}(\varepsilon)}{W^{(n q)}(b+\varepsilon)}\right) \\
= & \sum_{i=1}^{n}\left(\begin{array}{c}
n \\
i
\end{array}\right) \int_{0}^{\infty} z^{i} \int_{0}^{b+\varepsilon} u^{(n q)}(\varepsilon, y) \Pi(-y-\mathrm{d} z) \mathrm{d} y \ell_{n-i}(0, b) \\
& +n \varepsilon \ell_{n-1}(0, b)+o(\varepsilon) .
\end{aligned}
$$

Dividing both sides by $\varepsilon$, taking the limit as $\varepsilon \rightarrow 0$, and applying properties (P3) and (P4), and (2.3) yields (3.6).

\subsection{Positive initial reserve $0<\boldsymbol{u}<\boldsymbol{b}$}

Next, we obtain $\ell_{n}(u, b)$ for $0<u<b$.

Proposition 3.3. For $0 \leq u<b$,

$$
\begin{gathered}
\ell_{n}(u, b)=\sum_{i=1}^{n}\left(\begin{array}{c}
n \\
i
\end{array}\right) \int_{0}^{\infty} z^{i} \int_{0}^{b} u^{(n q)}(u, y) \Pi(-y-\mathrm{d} z) \mathrm{d} y \ell_{n-i}(0, b) \\
+\left(Z^{(n q)}(u)-W^{(n q)}(u) \frac{Z^{(n q)}(b)}{W^{(n q)}(b)}\right) \ell_{n}(0, b),
\end{gathered}
$$

where $u^{(n q)}(u, y)$ is given by (2.7).

Proof. We have

$$
\begin{aligned}
\ell_{n}(u, b)= & \mathbb{E}_{u}\left[\mathrm{e}^{-n q T_{(0, b)}} \mathbf{1}_{\left\{X_{T_{(0, b)}}<0\right\}}\left(\left|X_{T_{0}^{-}}\right|+L^{b}(0)\right)^{n}+\mathrm{e}^{\left.-n q T_{(0, b)}\right)} \mathbf{1}_{\left\{X_{T_{(0, b)}}=0\right\}}\left(L^{b}(0)\right)^{n}\right] \\
= & \mathbb{E}_{u}\left[\sum_{i=0}^{n} \mathrm{e}^{-n q T_{(0, b)}} \mathbf{1}_{\left\{X_{\left.T_{(0, b)}<0\right\}}\left(\begin{array}{c}
n \\
i
\end{array}\right)\left(\left|X_{T_{0}^{-}}\right|\right)^{i}\left(L^{b}(0)\right)^{n-i}\right]}\right] \\
& +\mathbb{E}_{u}\left[\mathrm{e}^{-n q T_{(0, b)}} \mathbf{1}_{\left\{X_{T_{(0, b)}} \leq 0\right\}}\left(L^{b}(0)\right)^{n}\right] \\
= & \sum_{i=1}^{n}\left(\begin{array}{c}
n \\
i
\end{array}\right) \int_{0}^{\infty} z^{i} \int_{0}^{b} u^{(n q)}(u, y) \Pi(-y-\mathrm{d} z) \mathrm{d} y \ell_{n-i}(0, b) \\
& \quad+\left(Z^{(n q)}(u)-W^{(n q)}(u) \frac{Z^{(n q)}(b)}{W^{(n q)}(b)}\right) \ell_{n}(0, b) .
\end{aligned}
$$

We applied the strong Markov property, (2.5), and (2.7) in the last equality.

\subsection{Moments of the discounted loss with recovery and no barrier}

Let

$$
L=\int_{0}^{\infty} \mathrm{e}^{-q t} \mathrm{~d} I_{t} .
$$

Let $\ell_{n}(u)=\mathbb{E}_{u}\left[L^{n}\right]$. Since $L^{b}(u)$ is monotone in $b, \ell_{n}(u)=\lim _{b \rightarrow \infty} \ell_{n}(u, b)$. 
Proposition 3.4. We have

$$
\begin{gathered}
\ell_{n}(0)=\frac{\Phi(n q)}{n q} \sum_{i=1}^{n}\left(\begin{array}{l}
n \\
i
\end{array}\right) \int_{z=0}^{\infty} z^{i} \int_{y=0}^{\infty} \mathrm{e}^{-\Phi(n q) y} \Pi(-y-\mathrm{d} z) \mathrm{d} y \ell_{n-i}(0) \\
+\frac{\sigma^{2}}{2} \frac{\Phi(n q)}{q} \ell_{n-1}(0) .
\end{gathered}
$$

For $u>0$,

$$
\begin{gathered}
\ell_{n}(u)=\frac{\Phi(n q)}{n q} \sum_{i=1}^{n}\left(\begin{array}{c}
n \\
i
\end{array}\right) \int_{z=0}^{\infty} z^{i} \int_{y=0}^{\infty}\left(W^{(n q)}(u) \mathrm{e}^{-\Phi(n q) y}-W^{(n q)}(u-y)\right) \\
\times \Pi(-y-\mathrm{d} z) \mathrm{d} y \ell_{n-i}(0) \\
+\left(Z^{(n q)}(u)-\frac{n q}{\Phi(n q)} W^{(n q)}(u)\right) \ell_{n}(0)
\end{gathered}
$$

Note that in the bounded variation case, $\sigma=0$ in (3.22).

Proof. First we prove (3.22). Since $W^{(q)}(x)=0$ for $x<0$, (3.6) can be written as

$$
\begin{gathered}
\ell_{n}(0, b)=\frac{1}{Z^{(n q)}(b)} \sum_{i=1}^{n}\left(\begin{array}{c}
n \\
i
\end{array}\right) \int_{z=0}^{\infty} z^{i} \int_{y=0}^{\infty} W^{(n q)}(b-y) \Pi(-y-\mathrm{d} z) \mathrm{d} y \ell_{n-i}(0, b) \\
+n \frac{\sigma^{2}}{2} \frac{W^{(n q)}(b)}{Z^{(n q)}(b)} \ell_{n-1}(0, b)
\end{gathered}
$$

Note that

$$
\frac{W^{(n q)}(b-y)}{Z^{(n q)}(b)}=\frac{W^{(n q)}(b-y)}{W^{(n q)}(b)} \frac{W^{(n q)}(b)}{Z^{(n q)}(b)},
$$

and

$$
\frac{W^{(n q)}(b-y)}{W^{(n q)}(b)}=\mathbb{E}_{b}\left(\mathrm{e}^{-n q T_{b}^{+}} \mathbf{1}_{\left\{T_{b+y}^{+}<T_{y}^{-}\right\}}\right) .
$$

is increasing in $b$. This can be deduced also from the following identity:

$$
\frac{W^{(n q)}(b-y)}{W^{(n q)}(b)}=\exp \left(-\int_{0}^{y} n(\bar{\varepsilon}>t+b-y) \mathrm{d} t\right),
$$

where $n$ is a measure on the space of excursions from the maximum, and $\bar{\varepsilon}$ is the maximal excursion height; see Bertoin (1996, Chapter 7). The following limit holds:

$$
\lim _{b \rightarrow \infty} \frac{W^{(n q)}(b-y)}{W^{(n q)}(b)}=\mathrm{e}^{-\phi(n q) y} ;
$$

see Kuznetsov et al. (2012, Lemma 3.3) or Hubalek and Kyprianou (2011, Section 3). For a given $x, 0<x<b$,

$$
\mathbb{E}_{x}\left[\mathrm{e}^{-n q T_{0}^{-}} \mathbf{1}_{\left\{T_{0}^{-}<T_{b}^{+}\right\}}\right]=Z^{(n q)}(x)-W^{(n q)}(x) \frac{Z^{(n q)}(b)}{W^{(n q)}(b)}
$$


is increasing in $b$, thus $W^{(n q)}(b) / Z^{(n q)}(b)$ is increasing in $b$. By Kuznetsov et al. (2012, Lemma 3.3),

$$
\lim _{b \rightarrow \infty} \frac{W^{(n q)}(b)}{Z^{(n q)}(b)}=\frac{\Phi(n q)}{n q} .
$$

Taking the limit as $b \rightarrow \infty$ in (3.24) and applying the monotone convergence theorem, we obtain (3.22). Similarly, taking the limit as $b \rightarrow \infty$ in (3.21), and substituting in (2.7), (3.22), (3.25), and (3.26), we obtain (3.23).

\section{Expected discounted loss with dividends and recovery}

In this section we consider the following risk process. Whenever ruin occurs the deficit is immediately recovered, and whenever the process reaches $b$ the overflow is paid as dividends. We consider the moments of the discounted loss. Let $\mathbb{L}^{b}(u)$ be the discounted loss given that $X_{0}=u$, and let $\mathcal{L}_{n}(u, b)$ be its $n$th moment. Let $D_{t}=\sup _{0 \leq s \leq t}\left(\left(X_{s}-b\right)_{+}\right)$, where $x_{+}=\max (x, 0)$ and $D_{t}$ is the dividends paid up to time $t$. Let $\tilde{U}_{t}=X_{t}-D_{t}$ and $U_{t}=$ $b-\tilde{U}_{t}$. Note that $U_{t}$ is the reflected process at its maximum. Throughout, we denote by $\hat{\tau}_{b}=\inf \left\{t: U_{t} \geq b\right\}=\inf \left\{t: \tilde{U}_{t} \leq 0\right\}$.

\subsection{Moments of the discounted loss-the bounded variation case}

Proposition 4.1. The following recursion holds:

$$
\begin{aligned}
\mathscr{L}_{n}(0, b)=\frac{W_{+}^{(n q)^{\prime}}(b)}{n q\left(W^{(n q)}(b)\right)^{2}}( & \sum_{i=1}^{n}\left(\begin{array}{l}
n \\
i
\end{array}\right) \int_{0}^{\infty} z^{i} \int_{0}^{b} W^{(n q)}(b-y) \Pi(-y-\mathrm{d} z) \mathrm{d} y \mathcal{L}_{n-i}(0, b) \\
& +\sum_{i=1}^{n}\left(\begin{array}{l}
n \\
i
\end{array}\right)\left(\int_{0}^{\infty} z^{i} \int_{0^{+}}^{b} \hat{r}^{(n q)}(0, y) \Pi(-(b-y)-\mathrm{d} z) \mathrm{d} y\right. \\
& \left.\left.+\int_{0}^{\infty} z^{i} \hat{r}^{(n q)}(0,0) \Pi(-b-\mathrm{d} z)\right) \mathcal{L}_{n-i}(0, b)\right) .
\end{aligned}
$$

Note that in the bounded variation case $\hat{r}^{(n q)}(0,0)>0$. Since Assumption 3.1, holds the right-hand side of (4.1) as well as similar expressions in the rest of this section are finite.

Proof. First we consider $\mathbb{L}^{b}(0)$,

$$
\mathbb{L}^{b}(0)=\mathbb{L}_{1}^{b}+\mathbb{L}_{2}^{b}
$$

where $\mathbb{L}_{1}^{b}=\mathbf{1}_{\left\{T_{0}^{-}<T_{b}^{+}\right\}} \mathrm{e}^{-q T_{0}^{-}}\left(\left|X_{T_{0}^{-}}\right|+\mathbb{L}^{b}(0)\right)$, and

$$
\mathbb{L}_{2}^{b}=\mathbf{1}_{\left\{T_{b}^{+}<T_{0}^{-}\right\}} \mathrm{e}^{-q T_{b}^{+}} \mathrm{e}^{-q \hat{\tau}_{b}}\left(\left(U_{\hat{\tau}_{b}}-b\right)+\mathbb{L}^{b}(0)\right) .
$$

Recall that $\hat{\tau}_{b}$ is the time the process $U$ hits $b$, or the time the process $\tilde{U}$ down-crosses 0 . Thus, given that $X_{0}=0, T_{b}^{+}+\hat{\tau}_{b}$ is the time the process down-crosses 0 when $T_{b}^{+}<T_{0}^{-}$, and $\left(U_{\hat{\tau}_{b}}-b\right)$ is the deficit.

By the strong Markov property, $T_{b}^{+}$and $\hat{\tau}_{b}$ are independent and independent of $\mathbb{L}^{b}(0)$.

Since $\mathbf{1}_{\left\{T_{0}^{-}<T_{b}^{+}\right\}} \mathbf{1}_{\left\{T_{b}^{+}<T_{0}^{-}\right\}}=0$, we have $\left(\mathbb{L}^{b}(0)\right)^{n}=\left(\mathbb{L}_{1}^{b}\right)^{n}+\left(\mathbb{L}_{2}^{b}\right)^{n}$ and

$$
\mathcal{L}_{n}(0, b)=\mathbb{E}_{0}\left[\left(\mathbb{L}_{1}^{b}\right)^{n}\right]+\mathbb{E}_{0}\left[\left(\mathbb{L}_{2}^{b}\right)^{n}\right] .
$$


Similar to (3.4),

$$
\mathbb{E}_{0}\left[\left(\mathbb{L}_{1}^{b}\right)^{n}\right]=\sum_{i=0}^{n}\left(\begin{array}{l}
n \\
i
\end{array}\right) \int_{z=0}^{\infty} z^{i} \int_{y=0}^{b} \frac{W^{(n q)}(0) W^{(n q)}(b-y)}{W^{(n q)}(b)} \Pi(-y-\mathrm{d} z) \mathrm{d} y \mathcal{L}_{n-i}(0, b) .
$$

Consider now $\mathbb{E}_{0}\left[\left(\mathbb{L}_{2}^{b}\right)^{n}\right]$. The strong Markov property implies that

$$
\mathbb{E}_{0}\left[\left(\mathbb{L}_{2}^{b}\right)^{n}\right]=\mathbb{E}_{0}\left[\mathrm{e}^{-n q T_{b}^{+}} \mathbf{1}_{\left\{T_{b}^{+}<T_{0}^{-}\right\}}\right] \mathbb{E}_{0}\left[\mathrm{e}^{-n q \hat{\tau}_{b}}\left(U_{\hat{\tau}_{b}}-b\right)+\mathbb{L}^{b}(0)^{n}\right] .
$$

Applying the strong Markov property again, we have

$$
\begin{aligned}
\mathbb{E}_{0}\left[\mathrm{e}^{-n q \hat{\tau}_{b}}\left(\left(U_{\hat{\tau}_{b}}-b\right)+\mathbb{L}^{b}(0)\right)^{n}\right] & =\mathbb{E}_{0}\left[\sum_{i=0}^{n} \mathrm{e}^{-n q \hat{\tau}_{b}}\left(\begin{array}{c}
n \\
i
\end{array}\right)\left(U_{\hat{\tau}_{b}}-b\right)^{i}\left(\mathbb{L}^{b}(0)\right)^{n-i}\right] \\
& =\sum_{i=0}^{n} \mathbb{E}_{0}\left[\mathrm{e}^{-n q \hat{\tau}_{b}}\left(\begin{array}{c}
n \\
i
\end{array}\right)\left(U_{\hat{\tau}_{b}}-b\right)^{i}\right] \mathcal{L}_{n-i}(0, b) .
\end{aligned}
$$

Applying (2.11), and (2.12) of Theorem 2.3 yields

$$
\begin{aligned}
\sum_{i=0}^{n} \mathbb{E}_{0}\left[\mathrm{e}^{-n q \hat{\tau}_{b}}\left(\begin{array}{c}
n \\
i
\end{array}\right)\left(U_{\hat{\tau}_{b}}-b\right)^{i}\right] \mathcal{L}_{n-i}(0, b) \\
=\sum_{i=0}^{n}\left(\begin{array}{c}
n \\
i
\end{array}\right)\left(\int_{0}^{\infty} z^{i} \int_{0^{+}}^{b} \hat{r}^{(n q)}(0, y) \Pi(-(b-y)-\mathrm{d} z) \mathrm{d} y\right. \\
\left.\quad+\int_{0}^{\infty} z^{i} \hat{r}^{(n q)}(0,0) \Pi(-b-\mathrm{d} z)\right) \mathcal{L}_{n-i}(0, b),
\end{aligned}
$$

where

$$
\hat{r}^{(n q)}(0, y)=W^{(n q)}(b) \frac{W_{+}^{(n q)^{\prime}}(y)}{W_{+}^{(n q)^{\prime}}(b)}-W^{(n q)}(y), \quad \hat{r}^{(n q)}(0,0)=W^{(n q)}(b) \frac{W^{(n q)}(0)}{W_{+}^{(n q)^{\prime}}(b)} .
$$

By Theorem 2.1(i),

$$
\mathbb{E}\left[\mathrm{e}^{-n q T_{b}^{+}} \mathbf{1}_{\left\{T_{b}^{+}<T_{0}^{-}\right\}}\right]=\frac{W^{(n q)}(0)}{W^{(n q)}(b)}=\frac{1}{c W^{(n q)}(b)} .
$$

From (4.2)-(4.4), we conclude that

$$
\begin{aligned}
\mathscr{L}_{n}(0, b)=\sum_{i=0}^{n}\left(\begin{array}{c}
n \\
i
\end{array}\right) \int_{0}^{\infty} z^{i} \int_{0}^{b} u^{(n q)}(0, y) \Pi(-y-\mathrm{d} z) \mathrm{d} y \mathcal{L}_{n-i}(0, b) \\
+\frac{W^{(n q)}(0)}{W^{(n q)}(b)} \sum_{i=0}^{n}\left(\begin{array}{c}
n \\
i
\end{array}\right)\left(\int_{0}^{\infty} z^{i} \int_{0^{+}}^{b} \hat{r}^{(n q)}(0, y) \Pi(-(b-y)-\mathrm{d} z) \mathrm{d} y\right. \\
\left.\quad+\int_{0}^{\infty} z^{i} \hat{r}^{(n q)}(0,0) \Pi(-b-\mathrm{d} z)\right) \mathcal{L}_{n-i}(0, b) .
\end{aligned}
$$


Note that, by (2.5),

$$
\begin{aligned}
\int_{0}^{\infty} \int_{0}^{b} u^{(n q)}(0, y) \Pi(-y-\mathrm{d} z) \mathrm{d} y & =\mathbb{E}_{0}\left[\mathrm{e}^{-n q T_{0}^{-}} \mathbf{1}_{\left\{X_{T_{(0, b)}} \leq 0\right\}}\right] \\
& =Z^{(n q)}(0)-W^{(n q)}(0) \frac{Z^{(n q)}(b)}{W^{(n q)}(b)} .
\end{aligned}
$$

Similarly, by (2.10),

$$
\begin{aligned}
& \int_{0^{+}}^{b} \int_{0}^{\infty} \hat{r}^{(n q)}(0, y) \Pi(-(b-y)-\mathrm{d} z) \mathrm{d} y+\int_{0}^{\infty} \hat{r}^{(n q)}(0,0) \Pi(-b-\mathrm{d} z) \\
& \quad=\mathbb{E}_{0}\left[\mathrm{e}^{-n q \hat{\tau}_{b}}\right] \\
& \quad=Z^{(n q)}(b)-\frac{n q\left(W^{(n q)}(b)\right)^{2}}{W_{+}^{(n q)^{\prime}}(b)} .
\end{aligned}
$$

By applying (4.5)-(4.7) and some simple algebra, the following expression is obtained for $\mathcal{L}_{n}(0, b)$ :

$$
\begin{aligned}
& \mathcal{L}_{n}(0, b)=\frac{W_{+}^{(n q)^{\prime}}(b)}{n q W^{(n q)}(b) W^{(n q)}(0)} \\
& \times\left(\sum_{i=1}^{n}\left(\begin{array}{l}
n \\
i
\end{array}\right) \int_{0}^{\infty} z^{i} \int_{0}^{b} u^{(n q)}(0, y) \Pi(-y-\mathrm{d} z) \mathrm{d} y \mathcal{L}_{n-i}(0, b)\right. \\
& +\frac{W^{(n q)}(0)}{W^{(n q)}(b)} \sum_{i=1}^{n}\left(\begin{array}{l}
n \\
i
\end{array}\right)\left(\int_{0^{+}}^{b} \int_{0}^{\infty} z^{i} \hat{r}^{(n q)}(0, y) \Pi(-(b-y)-\mathrm{d} z) \mathrm{d} y\right. \\
& \left.+\int_{0}^{\infty} z^{i} \hat{r}^{(n q)}(0,0) \Pi(-b-\mathrm{d} z)\right) \\
& \left.\times \mathcal{L}_{n-i}(0, b)\right) .
\end{aligned}
$$

Equation (4.1) is obtained by substituting the expression for $u^{(n q)}$ as in (2.7).

\subsection{The moments of discounted loss-the unbounded variation case $(\sigma>0)$}

Proposition 4.2. The following recursion holds:

$$
\begin{aligned}
\mathcal{L}_{n}(0, b)= & \frac{W^{(n q)^{\prime}}(b)}{n q\left(W^{(n q)}(b)\right)^{2}} \\
& \times\left(\sum_{i=1}^{n}\left(\begin{array}{c}
n \\
i
\end{array}\right) \int_{0}^{\infty} z^{i} \int_{0}^{b} W^{(n q)}(b-y) \Pi(-y-\mathrm{d} z) \mathrm{d} y \mathcal{L}_{n-i}(0, b)\right. \\
& \left.+\sum_{i=1}^{n}\left(\begin{array}{c}
n \\
i
\end{array}\right) \int_{0}^{\infty} z^{i} \int_{0^{+}}^{b} \hat{r}^{(n q)}(0, y) \Pi(-(b-y)-\mathrm{d} z) \mathrm{d} y \mathcal{L}_{n-i}(0, b)\right) \\
+ & \frac{\sigma^{2}}{2 q} \frac{W^{(n q)^{\prime}}(b)}{W^{(n q)}(b)} \mathcal{L}_{n-1}(0, b) .
\end{aligned}
$$


Proof. We consider lower and upper bounds for $\mathcal{L}_{n}(0, b)$. First consider a lower bound. Assume that the first recovery occurs at time $T_{-\varepsilon}^{-}$when $T_{-\varepsilon}^{-}<T_{b}^{+}$, and at time $T_{b}^{+}+\hat{\tau}_{b}$ if $T_{b}^{+}<T_{-\varepsilon}^{-}$. From that point onwards recovery occurs whenever the process down-crosses or hits 0 . Let $\underline{\mathbb{L}}^{b, \varepsilon}$ be the total discounted recovery under this policy. Since on $\left(T_{-\varepsilon}^{-}<T_{b}^{+}\right)$, the deficit is discounted only from time $T_{-\varepsilon}^{-}, \underline{\mathbb{L}}^{b, \varepsilon} \leq \mathbb{L}^{b}(0)$. Then

$$
\underline{\mathbb{L}}^{b, \varepsilon}=\mathbb{L}_{1}^{b, \varepsilon}+\underline{\mathbb{L}}_{2}^{b, \varepsilon}+\mathbb{L}_{3}^{b, \varepsilon}+\mathbb{L}_{4}^{b, \varepsilon},
$$

where

$$
\begin{aligned}
\mathbb{L}_{1}^{b, \varepsilon} & =\mathrm{e}^{-q T_{-\varepsilon}^{-}} \mathbf{1}_{\left\{T_{-\varepsilon}^{-}<T_{b}^{+}, X_{\left.T_{-\varepsilon}^{-}<-\varepsilon\right\}}\right.}\left(\left|X_{T_{-\varepsilon}^{-}}\right|+\mathbb{L}^{b}(0)\right), \\
\underline{\mathbb{L}}_{2}^{b, \varepsilon} & =\mathrm{e}^{-q T_{-\varepsilon}^{-}} \mathbf{1}_{\left\{T_{-\varepsilon}^{-}<T_{b}^{+}, X_{T_{-\varepsilon}^{-}}=-\varepsilon\right\}}\left(\varepsilon+\mathbb{L}^{b}(0)\right), \\
\mathbb{L}_{3}^{b, \varepsilon} & =\mathrm{e}^{-q T_{b}^{+}} \mathbf{1}_{\left\{T_{b}^{+}<T_{-\varepsilon}^{-}\right\}} \mathrm{e}^{-q \hat{\tau}_{b}} \mathbf{1}_{\left\{U_{\hat{\tau}_{b}}-b>0\right\}}\left(\left(U_{\hat{\tau}_{b}}-b\right)+\mathbb{L}^{b}(0)\right), \\
\mathbb{L}_{4}^{b, \varepsilon} & =\mathrm{e}^{-q T_{b}^{+}} \mathbf{1}_{\left\{T_{b}^{+}<T_{-\varepsilon}^{-}\right\}} \mathrm{e}^{-q \hat{\tau}_{b}} \mathbf{1}_{\left\{U_{\hat{\tau}_{b}}-b=0\right\}} \mathbb{L}^{b}(0), \\
\left(\underline{\mathbb{L}}^{b, \varepsilon}\right)^{n} & =\left(\mathbb{L}_{1}^{b, \varepsilon}\right)^{n}+\left(\underline{\mathbb{L}}_{2}^{b, \varepsilon}\right)^{n}+\left(\mathbb{L}_{3}^{b, \varepsilon}\right)^{n}+\left(\mathbb{L}_{4}^{b, \varepsilon}\right)^{n} .
\end{aligned}
$$

From (2.6) and (2.7), it follows that

$$
\mathbb{E}\left[\left(\mathbb{L}_{1}^{b, \varepsilon}\right)^{n}\right]=\sum_{i=0}^{n}\left(\begin{array}{l}
n \\
i
\end{array}\right) \int_{0}^{\infty} z^{i} \int_{0}^{b+\varepsilon} u^{(n q)}(\varepsilon, y) \Pi(-y-\mathrm{d} z) \mathrm{d} y \mathcal{L}_{n-i}(0, b) .
$$

Similarly, (2.4), (2.11), and (2.12) imply that

$$
\begin{aligned}
& \mathbb{E}\left[\left(\mathbb{L}_{3}^{b, \varepsilon}\right)^{n}\right] \\
& \quad=\frac{W^{(n q)}(\varepsilon)}{W^{(n q)}(b+\varepsilon)} \sum_{i=0}^{n}\left(\begin{array}{c}
n \\
i
\end{array}\right) \int_{0}^{\infty} z^{i} \int_{0}^{b} \hat{r}^{(n q)}(0, y) \Pi(-(b-y)-\mathrm{d} z) \mathrm{d} y \mathcal{L}_{n-i}(0, b) .
\end{aligned}
$$

Applying the strong Markov property, (3.12), and (3.13), it follows that

$$
\begin{aligned}
\mathbb{E}\left[\left(\underline{\mathbb{L}}_{2}^{b, \varepsilon}\right)^{n}\right] & =\mathbb{E}\left[\mathrm{e}^{-n q T_{-\varepsilon}^{-}} \mathbf{1}_{\left\{T_{-\varepsilon}^{-}<T_{b}^{+}, X_{T_{-\varepsilon}^{-}}=-\varepsilon\right\}}\right] \mathbb{E}\left[\left(\varepsilon+\mathbb{L}^{b}(0)\right)^{n}\right] \\
& =\frac{\sigma^{2}}{2}\left(W^{(n q)^{\prime}}(\varepsilon)-\frac{W^{(n q)^{\prime}}(b)}{W^{(n q)}(b)} W^{(n q)}(\varepsilon)\right) \mathcal{L}_{n}(0, b)+n \varepsilon \mathcal{L}_{n-1}(0, b)+o(\varepsilon) .
\end{aligned}
$$

The strong Markov property and (2.4) imply that

$$
\begin{aligned}
\mathbb{E}\left[\left(\mathbb{L}_{4}^{b, \varepsilon}\right)^{n}\right] & =\mathbb{E}_{0}\left[\mathrm{e}^{-n q T_{b}^{+}} \mathbf{1}_{\left\{T_{b}^{+}<T_{-\varepsilon}^{-}\right\}}\right] \mathbb{E}_{0}\left[\mathrm{e}^{-n q \hat{\tau}_{b}} \mathbf{1}_{\left\{U_{\hat{\tau}_{b}}-b=0\right\}}\right] \mathcal{L}_{n}(0, b) \\
& =\frac{W^{(n q)}(\varepsilon)}{W^{(n q)}(b+\varepsilon)} \mathbb{E}_{0}\left[\mathrm{e}^{-n q \hat{\tau}_{b}} \mathbf{1}_{\left\{U_{\hat{\tau}_{b}}-b=0\right\}}\right] \mathcal{L}_{n}(0, b) .
\end{aligned}
$$


Equations (4.9)-(4.13) imply that

$$
\begin{aligned}
\mathcal{L}_{n}(0, b) \geq & \sum_{i=0}^{n}\left(\begin{array}{c}
n \\
i
\end{array}\right) \int_{0}^{\infty} z^{i} \int_{0}^{b+\varepsilon} u^{(n q)}(\varepsilon, y) \Pi(-y-\mathrm{d} z) \mathrm{d} y \mathcal{L}_{n-i}(0, b) \\
& +\frac{\sigma^{2}}{2}\left(W^{(n q)^{\prime}}(\varepsilon)-\frac{W^{(n q)^{\prime}}(b)}{W^{(n q)}(b)} W^{(n q)}(\varepsilon)\right) \mathcal{L}_{n}(0, b)+n \varepsilon \mathcal{L}_{n-1}(0, b)+o(\varepsilon) \\
& +\frac{W^{(n q)}(\varepsilon)}{W^{(n q)}(b+\varepsilon)} \sum_{i=0}^{n}\left(\begin{array}{c}
n \\
i
\end{array}\right) \int_{0}^{b} \int_{0}^{\infty} z^{i} \hat{r}^{(n q)}(0, b-y) \Pi(-y-\mathrm{d} z) \mathrm{d} y \mathcal{L}_{n-i}(0, b) \\
& +\frac{W^{(n q)}(\varepsilon)}{W^{(n q)}(b+\varepsilon)} \mathbb{E}_{0}\left[\mathrm{e}^{-n q \hat{\tau}_{b}} \mathbf{1}_{\left\{U_{\hat{\tau}_{b}}-b=0\right\}}\right] \mathcal{L}_{n}(0, b) .
\end{aligned}
$$

Next, we establish an upper bound. Suppose that an amount $\varepsilon$ is injected at time 0 . When the process down-crosses $-\varepsilon$ by a jump, the deficit is recovered and the process restarts from 0 . When the process reaches $-\varepsilon$ by creeping before hitting $b$, it restarts from 0 (without deficit payment). From that point, the deficit is recovered whenever it occurs. Let $\overline{\mathbb{L}}^{\varepsilon, b}$ be the amount of the discounted money injected into the system. Since an amount $\varepsilon$ is injected in advance, $\overline{\mathbb{L}}^{\varepsilon, b} \geq L^{b}(0)$. Then

$$
\overline{\mathbb{L}}^{\varepsilon, b}=\varepsilon+\mathbb{L}_{1}^{b, \varepsilon}+\overline{\mathbb{L}}_{2}^{b, \varepsilon}+\mathbb{L}_{3}^{b, \varepsilon}+\mathbb{L}_{4}^{b, \varepsilon},
$$

where

$$
\overline{\mathbb{L}}_{2}^{b, \varepsilon}=\mathrm{e}^{-q T_{(-\varepsilon, b)}} \mathbf{1}_{\left\{X_{T_{-\varepsilon, b}}=-\varepsilon\right\}} \mathbb{L}^{b}(0)
$$

Thus,

$$
\begin{aligned}
\left(\overline{\mathbb{L}}^{\varepsilon, b}\right)^{n} & =\sum_{i=0}^{n}\left(\begin{array}{l}
n \\
i
\end{array}\right) \varepsilon^{i}\left(\mathbb{L}_{1}^{b, \varepsilon}+\overline{\mathbb{L}}_{2}^{b, \varepsilon}+\mathbb{L}_{3}^{b, \varepsilon}+\mathbb{L}_{4}^{b, \varepsilon}\right)^{n-i} \\
& =\sum_{i=0}^{n}\left(\begin{array}{l}
n \\
i
\end{array}\right) \varepsilon^{i}\left(\left(\mathbb{L}_{1}^{b, \varepsilon}\right)^{n-i}+\left(\overline{\mathbb{L}}_{2}^{b, \varepsilon}\right)^{n-i}+\left(\mathbb{L}_{3}^{b, \varepsilon}\right)^{n-i}+\left(\mathbb{L}_{4}^{b, \varepsilon}\right)^{n-i}\right) .
\end{aligned}
$$

Note that when $\sigma>0$, properties (P3) and (P4) imply that $\varepsilon^{i} W^{(n q)}(\varepsilon)=o(\varepsilon)$ for $i \geq 1$. Thus, (4.10), (4.11), and (4.13) imply that $\varepsilon^{i}\left(\mathbb{E}\left[\mathbb{L}_{j}^{b, \varepsilon}\right]\right)^{n-i}=o(\varepsilon), i \geq 1, j=1, \overline{3}, 4$. Equations (2.8) and (4.16) imply that, for $j=1, \ldots, n$,

$$
\mathbb{E}\left[\left(\overline{\mathbb{L}}_{2}^{b, \varepsilon}\right)^{j}\right]=\frac{\sigma^{2}}{2}\left(W^{(j q)^{\prime}}(\varepsilon)-\frac{W^{(j q)^{\prime}}(b)}{W^{(j q)}(b)} W^{(j q)}(\varepsilon)\right) \mathcal{L}_{j}(0, b) .
$$

Equations (3.12), (3.13), and (4.15)-(4.18) yield

$$
\begin{aligned}
\mathcal{L}_{n}(0) \leq & \mathbb{E}\left[\left(L_{1}^{b, \varepsilon}\right)^{n}\right]+\mathbb{E}\left[\left(\mathbb{L}_{3}^{b, \varepsilon}\right)^{n}\right]+\mathbb{E}\left[\left(\mathbb{L}_{4}^{b, \varepsilon}\right)^{n}\right]+n \varepsilon \mathcal{L}_{n-1}(0, b) \\
& +\frac{\sigma^{2}}{2}\left(W^{(n q)^{\prime}}(\varepsilon)-\frac{W^{(n q)^{\prime}}(b)}{W^{(n q)}(b)} W^{(n q)}(\varepsilon)\right) \mathcal{L}_{n}(0, b)+o(\varepsilon) .
\end{aligned}
$$

Since the right-hand side of (4.14) and (4.19) are equal, we conclude that (4.14) holds as 
equality up to $o(\varepsilon)$. Applying (2.8) and then (2.5) yields

$$
\begin{aligned}
\int_{0}^{\infty} & \int_{0}^{b+\varepsilon} u^{(n q)}(\varepsilon, y) \Pi(-y-\mathrm{d} z) \mathrm{d} y+\frac{\sigma^{2}}{2}\left(W^{(n q)^{\prime}}(\varepsilon)-\frac{W^{(n q)^{\prime}}(b)}{W^{(n q)}(b)} W^{(n q)}(\varepsilon)\right) \\
& =\mathbb{E}\left[\mathrm{e}^{-n q T_{-\varepsilon}^{-}} \mathbf{1}_{\left\{T_{-\varepsilon}^{-}<T_{b}^{+}\right\}}\right] \\
& =Z^{(n q)}(\varepsilon)-Z^{(n q)}(b+\varepsilon) \frac{W^{(n q)}(\varepsilon)}{W^{(n q)}(b+\varepsilon)} .
\end{aligned}
$$

Equations (2.11), (2.12), and (2.10) imply that

$$
\begin{aligned}
\int_{0}^{\infty} & \int_{0}^{b} \hat{r}^{(n q)}(0, b-y) \Pi(-y-\mathrm{d} z) \mathrm{d} y+\mathbb{E}\left[\mathrm{e}^{-\hat{\tau}_{b}} \mathbf{1}_{\left\{U_{\hat{\tau}_{b}}=b\right\}}\right] \\
& =\mathbb{E}\left[\mathrm{e}^{-n q \hat{\tau}_{b}}\right] \\
& =Z^{(n q)}(b)-n q \frac{\left(W^{(n q)}(b)\right)^{2}}{W^{(n q)^{\prime}}(b)} .
\end{aligned}
$$

Substituting (3.13) and (4.20) into (4.14) (with equality) yields

$$
\begin{aligned}
& \left(1-Z^{(n q)}(\varepsilon)+\frac{W^{(n q)}(\varepsilon)}{W^{(n q)}(b+\varepsilon)}\left(Z^{(n q)}(b+\varepsilon)-Z^{(n q)}(b)\right)\right. \\
& \left.+n q \frac{W^{(n q)}(\varepsilon)}{W^{(n q)}(b+\varepsilon)} \frac{\left(W^{(n q)}(b)\right)^{2}}{W^{(n q)^{\prime}}(b)}\right) \mathcal{L}_{n}(0, b) \\
& =\sum_{i=1}^{n}\left(\begin{array}{l}
n \\
i
\end{array}\right) \int_{0}^{\infty} z^{i} \int_{0}^{b+\varepsilon} \frac{W^{(n q)}(\varepsilon) W^{(n q)}(b-y)}{W^{(n q)}(b)} \Pi(-y-\mathrm{d} z) \mathrm{d} y \mathcal{L}_{n-i}(0, b) \\
& \quad+\frac{W^{(n q)}(\varepsilon)}{W^{(n q)}(b+\varepsilon)} \sum_{i=1}^{n}\left(\begin{array}{c}
n \\
i
\end{array}\right) \int_{0}^{b} \int_{0}^{\infty} z^{i} \hat{r}^{(n q)}(0, b-y) \Pi(-y-\mathrm{d} z) \mathrm{d} y \mathcal{L}_{n-i}(0, b) \\
& \quad+n \varepsilon \mathcal{L}_{n-1}(0, b)+o(\varepsilon) .
\end{aligned}
$$

Note that

$$
\begin{array}{ll}
\lim _{\varepsilon \rightarrow 0} W^{(n q)}(x+\varepsilon)=W^{(n q)}(x) \quad \text { for } x \geq 0, & \lim _{\varepsilon \rightarrow 0} \frac{Z^{(n q)}(\varepsilon)-1}{\varepsilon}=W^{(n q)}(0)=0, \\
\lim _{\varepsilon \rightarrow 0} \frac{Z^{(n q)}(b+\varepsilon)-Z^{(n q)}(b)}{\varepsilon}=W^{(n q)}(b), & \lim _{\varepsilon \rightarrow 0} \frac{W^{(n q)}(\varepsilon)}{\varepsilon}=W^{(n q)^{\prime}}(0)=\frac{2}{\sigma^{2}} .
\end{array}
$$

Thus, dividing (4.21) by $\varepsilon$ and taking the limit as $\varepsilon \rightarrow 0$ yields

$$
\begin{aligned}
& n q W^{(n q)^{\prime}}(0) \frac{W^{(n q)}(b)}{W^{(n q)^{\prime}}(b)} \mathcal{L}_{n}(b, 0) \\
& =\frac{W^{(n q)^{\prime}}(0)}{W^{(n q)}(b)} \sum_{i=1}^{n}\left(\begin{array}{c}
n \\
i
\end{array}\right) \int_{0}^{\infty} z^{i} \int_{0}^{b} W^{(n q)}(b-y) \Pi(-y-\mathrm{d} z) \mathrm{d} y \mathcal{L}_{n-i}(0, b) \\
& +\frac{W^{(n q)^{\prime}}(0)}{W^{(n q)}(b)} \sum_{i=1}^{n}\left(\begin{array}{c}
n \\
i
\end{array}\right) \int_{0}^{b} \int_{0}^{\infty} z^{i} \hat{r}^{(n q)}(0, b-y) \mathrm{d} y \Pi(-y-\mathrm{d} z) \mathrm{d} y \mathcal{L}_{n-i}(0, b) \\
& \quad+n \mathcal{L}_{n-1}(0, b) .
\end{aligned}
$$


Since $W^{(n q)^{\prime}}(0)=2 / \sigma^{2}>0$, dividing both sides of (4.22) by

$$
n q W^{(n q)^{\prime}}(0) \frac{W^{(n q)}(b)}{W^{(n q)^{\prime}}(b)}
$$

yields (4.8).

Remark 4.1. Note that (4.1) and (4.8) are equivalent once we note that for the bounded variation case $\sigma=0$ and for the unbounded variation case $\hat{r}(0,0)=0$.

\subsection{Expected discounted moments when the initial reserve is greater than 0}

Assume that $X_{0}=x$ with $0<x<b$. Then

$$
\begin{aligned}
\mathcal{L}_{n}(x, b)= & \sum_{i=1}^{n}\left(\begin{array}{c}
n \\
i
\end{array}\right) \int_{0}^{\infty} z^{i} \int_{0}^{b} u^{(n q)}(x, y) \Pi(-y-\mathrm{d} z) \mathrm{d} y \mathcal{L}_{n-i}(0, b) \\
& +\frac{W^{(n q)}(x)}{W^{(n q)}(b)} \sum_{i=1}^{n}\left(\begin{array}{c}
n \\
i
\end{array}\right)\left(\int_{0}^{b} \int_{0}^{\infty} z^{i} \hat{r}^{(n q)}(b-x, b-y) \Pi(-y-\mathrm{d} z) \mathrm{d} y\right. \\
& \left.+\int_{0}^{\infty} z^{i} \hat{r}^{(n q)}(0,0) \Pi(-b-\mathrm{d} z)\right) \mathcal{L}_{n-i}(0, b) \\
& +\left(Z^{(n q)}(x)-n q \frac{W^{(n q)}(b) W^{(n q)}(x)}{W_{+}^{(n q)^{\prime}}(b)}\right) \mathcal{L}_{n}(0, b) .
\end{aligned}
$$

Proof. Similar arguments as in Sections 4.1 and 4.2 imply that

$$
\begin{aligned}
\mathscr{L}_{n}(x, b)= & \mathbb{E}_{x}\left[\left(\mathrm{e}^{-q T_{0}^{-}} \mathbf{1}_{\left\{T_{0}^{-}<T_{b}^{+}, X_{\left.T_{0}^{-}<0\right\}}\right.}\left(\left|X_{T_{0}^{-}}\right|+\mathbb{L}^{b}(0)\right)\right)^{n}\right] \\
& +\mathbb{E}_{x}\left[\left(\mathrm{e}^{-q T_{0}^{-}} \mathbf{1}_{\left\{T_{0}^{-}<T_{b}^{+}, X_{T_{0}^{-}}=0\right\}} \mathbb{L}^{b}(0)\right)^{n}\right] \\
& +\mathbb{E}_{x}\left[\left(\mathrm{e}^{-q T_{b}^{+}} \mathrm{e}^{-q \hat{\tau}_{b}} \mathbf{1}_{\left\{T_{b}^{+}<T_{0}^{-}\right\}} \mathbf{1}_{\left\{U_{\hat{\tau}_{b}}>b\right\}}\left(\left(U_{\hat{\tau}_{b}}-b\right)+\mathbb{L}^{b}(0)\right)\right)^{n}\right] \\
& +\mathbb{E}_{x}\left[\left(\mathrm{e}^{-q T_{b}^{+}} \mathrm{e}^{-q \hat{\tau}_{b}} \mathbf{1}_{\left\{T_{b}^{+}<T_{0}^{-}\right\}} \mathbf{1}_{\left\{U_{\hat{\tau}_{b}}=b\right\}} \mathbb{L}^{b}(0)\right)^{n}\right] .
\end{aligned}
$$

Expanding the above expressions, applying (2.4), (2.5), and (2.10), and using the same arguments as in the previous sections yields the result. Note that the last term in (4.23) is

$$
\mathbb{E}_{x}\left[\left(\mathrm{e}^{-n q T_{0}^{-}} \mathbf{1}_{\left\{T_{0}^{-}<T_{b}^{+}, X_{T_{0}^{-}} \leq 0\right\}}\right)+\left(\mathrm{e}^{-n q T_{b}^{+}} \mathrm{e}^{-n q \hat{\tau}_{b}} \mathbf{1}_{\left\{T_{b}^{+}<T_{0}^{-}\right\}} \mathbf{1}_{\left\{U_{\hat{\tau}_{b}} \geq b\right\}}\right)\right] \mathcal{L}_{n}(0, b) .
$$

\subsection{An example}

We consider a similar example as in Schmidli and Kulenko (2008). Consider a risk process with recoveries and dividend payments with the following parameters: claims arrive according to a Poisson process at rate $\lambda=2$, the premium rate is $c=4$, the claims amount are independent and identically distributed exponentially distributed with parameter $\alpha=3$, and the discounted factor is $q=0.06$. For this model Kulenko and Schmidli proved that the strategy that maximizes the expected discounted dividends is the barrier strategy with $b=1.271$. The $q$-scale function for this process is

$$
W^{(q)}(x)=\exp \frac{\left(x \xi_{2}\right)}{\psi^{\prime}\left(\xi_{2}\right)}+\exp \frac{\left(-x \xi_{1}\right)}{\psi^{\prime}\left(-\xi_{1}\right)},
$$



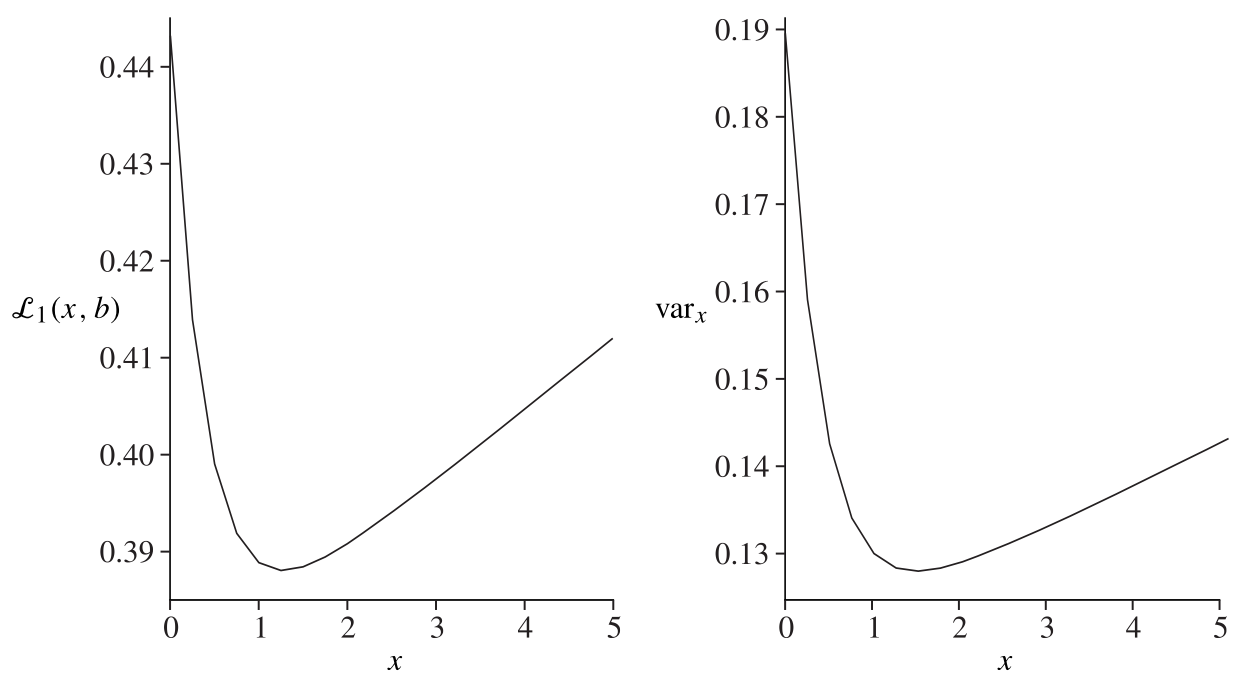

FIGURE 1: The expected discounted loss (left) and the variance of the discounted loss (right).

where $\psi(\theta)$ is the Laplace exponent of the process,

$$
\psi(\theta)=c \theta-\frac{\lambda \theta}{\alpha+\theta},
$$

and $\xi_{i}, i=1,2$, are the roots of the equations $\psi(\theta)=q$ and are given by

$$
\xi_{1}=\frac{\sqrt{(\lambda+q-c \alpha)^{2}+4 c q \alpha}-\lambda-q+c \alpha}{2 c}
$$

and

$$
\xi_{2}=\frac{\sqrt{(\lambda+q-c \alpha)^{2}+4 c q \alpha}+\lambda+q-c \alpha}{2 c} .
$$

Schmidli and Kulenko obtained the first moment of the discounted deficit. Using the recursion, we obtained the second moment and, hence, the variance of the discounted dividends $\operatorname{var}_{x}$ for the initial reserve $x$. In Figure 1 we show the expected discounted loss $\mathcal{L}_{1}(x, b)$ and the variance of the discounted loss as functions of the initial reserve $x$.

\section{Discounted dividend payments in a model with dividend barrier and recovery}

Consider a spectrally negative Lévy risk process with a dividend barrier $b$, where all the overflow above $b$ is paid as dividends. Let $\tilde{V}_{n}(u)$ be the $n$th moment of the discounted dividends until ruin when the initial reserve is $u, 0<u \leq b$. Renaud and Zhou (2007) and Kyprianou and Palmowski (2007) obtained the following formula for $\tilde{V}_{n}(u)$ :

$$
\tilde{V}_{n}(u)=\frac{W^{(n q)}(u)}{W^{(n q)}(b)} n ! \prod_{i=1}^{n} \frac{W^{(i q)}(b)}{W^{(i q)^{\prime}}(b)} .
$$

In this section we consider a risk model with recoveries and dividend payments according to the barrier strategy and compute the moments of the discounted dividends. We apply a similar method as in Renaud and Zhou (2007). Let $D_{t}$ be the dividends paid up to time $t$ and let $V(u)$ 
be the discounted dividends when the initial reserve is $u$. The $n$th moment of the discounted dividends given initial reserve $u$ is

$$
\mathcal{V}_{n}(u)=\mathbb{E}_{u}\left[\int_{0}^{\infty} \mathrm{e}^{-q t} \mathrm{~d} D_{t}\right]^{n} .
$$

When the initial reserve is $b$, then until $X_{t}-D_{t}$ down-crosses 0 , the process $b-\left(X_{t}-D_{t}\right)$ evolves as the process $\hat{Y}$-the reflection of $X_{t}$ at its maximum. Similarly, from the moment of recovery, the process evolves as $Y_{t}$-the reflection at the infimum. In this section we do not assume that Assumption 3.1 holds.

\subsection{Moments when the initial reserve is $b$}

First we obtain $\mathcal{V}_{n}(b)$.

Proposition 5.1. It holds that

$$
\mathcal{V}_{n}(b)=\frac{1}{q^{n}} \prod_{i=1}^{n} \frac{Z^{(i q)}(b)}{W^{(i q)}(b)} .
$$

Proof. First we construct a lower bound. Consider the following dividend strategy. If the process reaches $b+\varepsilon$ before down-crossing 0 , an amount of $\varepsilon$ is paid, the process restarts form $b$, and from that point onwards all the overflow above $b$ is paid as dividends. When the process down-crosses 0 before reaching $b+\varepsilon$, no dividends are paid until the process reaches $b$ again, and then all the overflow above $b$ is paid as dividends. Let $\underline{V}(b)$ be the discounted dividends under this policy. Since with positive probability dividend payments are delayed, it follows that

$$
\underline{V}(b) \leq V(b), \quad \underline{V}(b)=\mathrm{e}^{-q T_{b+\varepsilon}^{+}} \mathbf{1}_{\left\{T_{b+\varepsilon}^{+}<T_{0}^{-}\right\}}(\varepsilon+V(b))+\mathrm{e}^{-q T_{0}^{-}} \mathbf{1}_{\left\{T_{0}^{-}<T_{b+\varepsilon}^{+}\right\}} \mathrm{e}^{-q \tau_{b}} V(b) .
$$

Recall that $\tau_{b}$ is the time that the reflected process at its infimum hits $b$. Therefore,

$$
\begin{aligned}
(\underline{V}(b))^{n}= & \mathrm{e}^{-n q T_{b+\varepsilon}^{+}} \mathbf{1}_{\left\{T_{b+\varepsilon}^{+}<T_{0}^{-}\right\}}(\varepsilon+V(b))^{n}+\mathrm{e}^{-n q T_{0}^{-}} \mathbf{1}_{\left\{T_{0}^{-}<T_{b+\varepsilon}^{+}\right\}} \mathrm{e}^{-n q \tau_{b}}(V(b))^{n} \\
= & \mathrm{e}^{-n q T_{b+\varepsilon}^{+}} \mathbf{1}_{\left\{T_{b+\varepsilon}^{+}<T_{0}^{-}\right\}} \sum_{i=0}^{n}\left(\begin{array}{c}
n \\
i
\end{array}\right) \varepsilon^{i}(V(b))^{n-i} \\
& +\mathrm{e}^{-n q T_{0}^{-}} \mathbf{1}_{\left\{T_{0}^{-}<T_{b+\varepsilon}^{+}\right\}} \mathrm{e}^{-n q \tau_{b}}(V(b))^{n} .
\end{aligned}
$$

Taking expectations from both sides of (5.1), we obtain

$$
\begin{aligned}
\mathbb{E}_{b}\left[(\underline{V}(b))^{n}\right] \\
=\frac{W^{(n q)}(b)}{W^{(n q)}(b+\varepsilon)} \sum_{i=0}^{n}\left(\begin{array}{l}
n \\
i
\end{array}\right) \varepsilon^{i} \mathcal{V}_{n-i}(b) \\
\quad+\left(Z^{(n q)}(b)-Z^{(n q)}(b+\varepsilon) \frac{W^{(n q)}(b)}{W^{(n q)}(b+\varepsilon)}\right) \frac{Z^{(n q)}(0)}{Z^{(n q)}(b)} \mathcal{V}_{n}(b) \\
=\mathcal{V}_{n}(b)\left(\frac{W^{(n q)}(b)}{W^{(n q)}(b+\varepsilon)}+\left(Z^{(n q)}(b)-Z^{(n q)}(b+\varepsilon) \frac{W^{(n q)}(b)}{W^{(n q)}(b+\varepsilon)}\right) \frac{Z^{(n q)}(0)}{Z^{(n q)}(b)}\right) \\
\quad+n \varepsilon \frac{W^{(n q)}(b)}{W^{(n q)}(b+\varepsilon)} \mathcal{V}_{n-1}(b)+o(\varepsilon) .
\end{aligned}
$$

We applied (2.4) in the first line of (5.3) and (2.5) and (2.9) in the second line. 
To obtain an upper bound consider the following strategy. An amount $\varepsilon$ is paid at time 0 , and then the process restarts from $b$. No dividend is paid until time $T_{b+\varepsilon}^{+} \wedge T_{0}^{-}$. If $T_{b+\varepsilon}^{+}<T_{0}^{-}$, the process restarts from $b$ without dividend payment and from that point onwards all the overflow is paid as dividends. If $T_{0}^{-}<T_{b+\varepsilon}^{+}$, all the overflow above $b$ is paid as dividends. Let $\bar{V}(b)$ be the discounted dividends under this strategy. Since $\varepsilon$ is paid in advance $\bar{V}(b) \geq V(b)$, and

$$
(\bar{V}(b))^{n}=\left(\varepsilon+\mathrm{e}^{-q T_{b+\varepsilon}^{+}} \mathbf{1}_{\left\{T_{b+\varepsilon}^{+}<T_{0}^{-}\right\}} V(b)+\mathrm{e}^{-q T_{0}^{-}} \mathrm{e}^{-q \tau_{b}} \mathbf{1}_{\left\{T_{0}^{-}<T_{b+\varepsilon}^{+}\right\}} V(b)\right)^{n} .
$$

Thus,

$$
\begin{aligned}
(\bar{V}(b))^{n}=\sum_{i=0}^{n}\left(\begin{array}{l}
n \\
i
\end{array}\right) \varepsilon^{i}\left(\mathbf{1}_{\left\{T_{b+\varepsilon}^{+}<T_{0}^{-}\right\}} \mathrm{e}^{-(n-i) q T_{b+\varepsilon}^{+}}(V(b))^{n-i}\right. \\
\left.+\mathbf{1}_{\left\{T_{0}^{-}<T_{b+\varepsilon}^{+}\right\}} \mathrm{e}^{-(n-i) q T_{0}^{-}} \mathrm{e}^{-(n-i) q \tau_{b}}(V(b))^{n-i}\right)
\end{aligned}
$$

Taking expectations and applying (2.4), (2.5), and (2.9), we obtain

$$
\begin{aligned}
\mathbb{E}[\bar{V}(b)]^{n} & \\
=\left(\frac{W^{(n q)}(b)}{W^{(n q)}(b+\varepsilon)}+\left(Z^{(n q)}(b)\right.\right. & \left.\left.-Z^{(n q)}(b+\varepsilon) \frac{W^{(n q)}(b)}{W^{(n q)}(b+\varepsilon)}\right) \frac{Z^{(n q)}(0)}{Z^{(n q)}(b)}\right) \mathcal{V}_{n}(b) \\
+n \varepsilon\left(\frac{W^{((n-1) q)}(b)}{W^{((n-1) q)}(b+\varepsilon)}+(\right. & \left.Z^{((n-1) q)}(b)-Z^{((n-1) q)}(b+\varepsilon) \frac{W^{((n-1) q)}(b)}{W^{((n-1) q)}(b+\varepsilon)}\right) \\
& \left.\times \frac{Z^{((n-1) q)}(0)}{Z^{((n-1) q)}(b)}\right) \mathcal{V}_{n-1}(b)+o(\varepsilon) .
\end{aligned}
$$

Let

$$
A=\left(Z^{((n-1) q)}(b)-Z^{((n-1) q)}(b+\varepsilon) \frac{W^{((n-1) q)}(b)}{W^{((n-1) q)}(b+\varepsilon)}\right) \frac{Z^{((n-1) q)}(0)}{Z^{((n-1) q)}(b)} .
$$

After some calculation $A$ can be written as

$$
A=\frac{Z^{((n-1) q)}(b)\left(W^{((n-1) q)}(b+\varepsilon)-W^{((n-1) q)}(b)\right)-W^{((n-1) q)}(b)\left(Z^{((n-1) q)}(b+\varepsilon)-Z^{((n-1) q)}(b)\right)}{W^{((n-1) q)}(b+\varepsilon) Z^{((n-1) q)}(b)},
$$

Since $W^{(n-1) q}$ is differentiable from the right at $b$ (see Kuznetsov et al. (2012, Equation (32))) and $Z^{(n-1) q}$ is differentiable, where $Z^{((n-1) q)^{\prime}}(b)=(n-1) q W^{((n-1) q)}(b)$, the last expression can be written as follows:

$$
\begin{aligned}
A= & \frac{1}{W^{((n-1) q)}(b+\varepsilon) Z^{((n-1) q)}(b)} Z^{((n-1) q)}(b) W_{+}^{((n-1) q)^{\prime}}(b) \varepsilon-(n-1) q\left(W^{((n-1) q)}(b)\right)^{2} \varepsilon \\
& +o(\varepsilon) .
\end{aligned}
$$

Thus, $\varepsilon A=o(\varepsilon)$, and

$$
\begin{aligned}
\mathbb{E}[\bar{V}(b)]^{n}= & \left(\frac{W^{(n q)}(b)}{W^{(n q)}(b+\varepsilon)}+\left(Z^{(n q)}(b)-Z^{(n q)}(b+\varepsilon) \frac{W^{(n q)}(b)}{W^{(n q)}(b+\varepsilon)}\right) \frac{Z^{(n q)}(0)}{Z^{(n q)}(b)}\right) \mathcal{V}_{n}(b) \\
& +n \varepsilon \frac{W^{((n-1) q)}(b)}{W^{((n-1) q)}(b+\varepsilon)} \mathcal{V}_{n-1}(b)+o(\varepsilon)
\end{aligned}
$$


Equations (5.3) and (5.4) yield $\mathbb{E}[\bar{V}(b)]^{n}=\mathbb{E}[\underline{V}(b)]^{n}$ up to $o(\varepsilon)$, and, thus,

$$
\begin{aligned}
\mathcal{V}_{n}(b)= & \left(\frac{W^{(n q)}(b)}{W^{(n q)}(b+\varepsilon)}+\left(Z^{(n q)}(b)-Z^{(n q)}(b+\varepsilon) \frac{W^{(n q)}(b)}{W^{(n q)}(b+\varepsilon)}\right) \frac{1}{Z^{(n q)}(b)}\right) \mathcal{V}_{n}(b) \\
& +n \varepsilon \frac{W^{((n-1) q)}(b)}{W^{((n-1) q)}(b+\varepsilon)} \mathcal{V}_{n-1}(b)+o(\varepsilon) .
\end{aligned}
$$

Solving for $\mathcal{V}_{n}(b)$ yields

$$
\mathcal{V}_{n}(b)\left(\frac{W^{(n q)}(b)}{W^{(n q)}(b+\varepsilon)}\right)\left(\frac{Z^{(n q)}(b+\varepsilon)-Z^{(n q)}(b)}{Z^{(n q)}(b)}\right)=n \varepsilon \frac{W^{((n-1) q)}(b)}{W^{((n-1) q)}(b+\varepsilon)} \mathcal{V}_{n-1}(b)+o(\varepsilon) .
$$

Dividing by $\varepsilon$ and taking the limit as $\varepsilon$ goes to 0 , we obtain

$$
\mathcal{V}_{n}(b)=\frac{Z^{(n q)}(b)}{q W^{(n q)}(b)} \mathcal{V}_{n-1}(b)
$$

\subsection{Moments when the initial reserve is $u<b$}

Proposition 5.2. Let $0 \leq u \leq b$ then

$$
\mathcal{V}_{n}(u)=\left(\frac{W^{(n q)}(u)}{W^{(n q)}(b)}+\left(Z^{(n q)}(u)-Z^{(n q)}(b) \frac{W^{(n q)}(u)}{W^{(n q)}(b)}\right) \frac{1}{Z^{(n q)}(b)}\right) \mathcal{V}_{n}(b) .
$$

Proof. Starting at $u$, either $b$ is reached before recovery, or recovery occurs before reaching $b$. Applying the strong Markov property,

$$
\mathcal{V}_{n}(u)=\left(\mathbb{E}_{u}\left[\mathrm{e}^{-n q T_{b}^{+}} \mathbf{1}_{\left\{T_{b}^{+}<T_{0}^{-}\right\}}\right]+\mathbb{E}_{u}\left[\mathrm{e}^{-n q T_{0}^{-}} \mathbf{1}_{\left\{T_{0}^{-}<T_{b}^{+}\right\}}\right] \mathbb{E}\left[\mathrm{e}^{-n q \tau_{b}}\right]\right) \mathcal{V}_{n}(b) .
$$

The result follows by applying (2.4), (2.5), and (2.9).

\section{Acknowledgement}

I would like to thank the anonymous referee for the careful reading and useful comments and suggestions that improved the presentation of this paper.

\section{References}

[1] Asmussen, S. AND TAKsar, M. (1997). Controlled diffusion models for optimal dividend pay-out. Insurance Math. Econom. 20, 1-15.

[2] Asmussen, S., Højgaard, B. and Taksar, M. (2000). Optimal risk control and dividend distribution policies. Example of excess-of loss reinsurance for an insurance corporation. Finance Stoch. 4, 299-324.

[3] Avanzi, B. and Gerber, H. U. (2008) Optimal dividends in the dual model with diffusion. ASTIN Bull. 38, 653-667.

[4] Avanzi, B., Gerber, H. U. and Shiu, E. W. S. (2007). Optimal dividends in the dual model. Insurance Math. Econom. 41, 111-123.

[5] Avanzi, B., Shen, J. AND Wong, B. (2011). Optimal dividends and capital injections in the dual model with diffusion. ASTIN Bull. 41, 611-644.

[6] Avram, F., Palmowski, Z. and Pistorius, M. R. (2007). On the optimal dividend problem for a spectrally negative Lévy process. Ann. Appl. Prob. 17, 156-180.

[7] Bayraktar, E., Kyprianou, A. E. AND YamaZaKi, K. (2013). On optimal dividends in the dual model. ASTIN Bull. 43, 359-372.

[8] Bertoin, J. (1996). Lévy Processes. Cambridge University Press. 
[9] Bertorn, J. (1997). Exponential decay and ergodicity of completely asymmetric Lévy processes in a finite interval. Ann. Appl. Prob. 7, 156-169.

[10] Cheung, E. C. K. And Drekic, S. (2008). Dividend moments in the dual risk model: exact and approximate approaches. ASTIN Bull. 38, 399-422.

[11] Dickson, D. C. M. And Waters, H. R. (2004). Some optimal dividends problems. ASTIN Bull. 34, 49-74.

[12] De Finetti, B. (1957). Su un'impostazione alternativa dell teoria collettiva del rischio. Trans. XVth Internat. Congr. Actuaries 2, 433-443.

[13] Gerber, H. U. (1969). Entscheidungskriterien für den zusammengesetzten Poisson-prozess. Schweiz. Verein. Versicherungsmath. 69, 185-228.

[14] Hubalek, F. And Kyprianou, A. E. (2011). Old and new examples of scale functions for spectrally negative Lévy processes. In Seminar on Stochastic Analysis, Random Fields and Applications VI (Progress Prob. 63), Birkhäuser, Basel, pp. 119-145.

[15] Kulenko, N. And Schmidli, H. (2008). Optimal dividend strategies on a Cramér-Lundberg model with capital injections. Insurance Math. Econom. 43, 270-278.

[16] Kuznetsov, A., Kyprianou, A. E. And Rivero, V. (2012). The theory of scale functions for spectrally negative Lévy processes. In Lévy Matters II (Lecture Notes Math. 2061), Springer, Heidelberg, pp. 97-186.

[17] Kyprianou, A. E. (2006). Introductory Lectures on Fluctuations of Lévy Processes with Applications. Springer, Berlin.

[18] Kyprianou, A. E. And Palmowski, Z. (2007). Distributional study of de Finetti's dividend problem for a general Lévy insurance risk process. J. Appl. Prob. 44, 428-443.

[19] Loeffen, R. L. (2008). On optimality of the barrier strategy in de Finetti's dividend problem for spectrally negative Lévy processes. Ann. Appl. Prob. 18, 1669-1680.

[20] Mijatović, A. AND Pistorius, M. R. (2012). On the drawdown of completely asymmetric Lévy processes. Stoch. Process. Appl. 122, 3812-3836.

[21] Pistorius, M. R. (2004). On exit and ergodicity of the spectrally one-sided Lévy process reflected at its infimum. J. Theoret. Prob. 17, 183-220.

[22] Renaud, J-F. AND Zhou, X. (2007). Distribution of the present value of dividend payments in a Lévy risk model. J. Appl. Prob. 44, 420-427.

[23] SchmiduI, H. (2008). Stochastic Control in Insurance. Springer, London.

[24] Suprun, V. N. (1976). Problem of destruction and resolvent of a terminating process with independent increments. Ukrainian Math. J. 28, 39-51. 